\title{
Conformational change in the monomeric alpha-synuclein imparts fibril polymorphs
}

\section{Short title: The monomeric $\alpha$ Syn imparts fibril polymorphs}

Cesar Aguirre ${ }^{1, \dagger}$, Kensuke Ikenaka ${ }^{1 *, \dagger}$, Masatomo So $^{2}$, Takahiro Maruno ${ }^{3}$, Keiichi Yamaguchi ${ }^{4}$, Kichitaro Nakajima ${ }^{4}$, Chi-Jing Choong ${ }^{1}$, Kei Nabekura ${ }^{1}$, Goichi Beck ${ }^{1}$, Kentaro Tomii ${ }^{5}$, Yu Yamamori ${ }^{5}$, Junko Doi ${ }^{1}$, Tomoyasu Matsubara ${ }^{6}$, Maho Morishima ${ }^{6}$, Keita Kakuda ${ }^{1}$, Makoto Hideshima $^{1}$, Yasuyoshi Kimura ${ }^{1}$, Seiichi Nagano ${ }^{1}$, Kousuke Baba ${ }^{1}$, Shigeo Murayama ${ }^{6}{ }^{6}$, Hirotsugu $\mathrm{Ogi}^{8}$, Yoshitaka Nagai ${ }^{9}$, Yasushi Kawata ${ }^{10}$, Susumu Uchiyama ${ }^{3}$, Yohei Miyanoiri ${ }^{11}$, Yuji Goto ${ }^{4}$, and Hideki Mochizuki ${ }^{*}$

${ }^{1}$ Department of Neurology, Osaka University Graduate School of Medicine, 2-2 Yamadaoka, Suita, Osaka 5650871, Japan.

${ }^{2}$ Institute for Protein Research, Osaka University, Japan, 3-2 Yamadaoka, Suita, Osaka 565-0871, Japan.

${ }^{3}$ Department of Biotechnology, Graduate School of Engineering, Osaka University, Japan, 2-1 Yamadaoka, Suita, Osaka 565-0871, Japan.

${ }^{4}$ Global Center for Medical Engineering and Informatics, Osaka University, Japan, 2-1 Yamadaoka, Suita, Osaka 565-0871, Japan.

5 Artificial Intelligence Research Center (AIRC), National Institute of Advanced Industrial Science and Technology (AIST), 2-4-7 Aomi, Koto-ku, Tokyo, 135-0064, Japan.

${ }^{6}$ Department of Neuropathology (the Brain Bank for Aging Research), Tokyo Metropolitan Geriatric Hospital and Institute of Gerontology, 35-2 Sakae-cho, Itabashi-ku, Tokyo, 173-0015, Japan.

${ }^{7}$ Brain Bank for Neurodevelopmental, Neurological and Psychiatric Disorders, United Graduate School of Child Development, Osaka University, 2-2, Yamadaoka, Suita- shi, Osaka, 565-0871, Japan.

${ }^{8}$ Graduate School of Engineering, Osaka University, Suita, Osaka 565-0871, Japan, 2-1 Yamadaoka, Suita, Osaka 565-0871, Japan.

${ }^{9}$ Department of Neurology, Faculty of Medicine, Kindai University, Osaka-Sayama, Osaka, Japan.

${ }^{10}$ Department of Chemistry and Biotechnology, Graduate School of Engineering, Tottori University, Tottori 680-8552, Japan.

${ }^{11}$ Laboratory for Ultra-High magnetic field NMR spectroscopy, Research Center for Next-Generation Protein Sciences, Institute for Protein Research, Osaka University, Japan, 3-2 Yamadaoka, Suita, Osaka 565-0871, Japan.

*Correspondence to Dr. Kensuke Ikenaka, M.D, Ph.D., and Prof. Hideki Mochizuki, M.D., Ph.D.

Department of Neurology, Osaka University Graduate School of Medicine, 2-2 Yamadaoka, Osaka 565-0871, Japan. Phone: 81-6-6879-3571.

E-mail: ikenaka@neurol.med.osaka-u.ac.jp, hmochizuki@neurol.med.osaka-u.ac.jp

$\dagger$ These authors contributed equally to this work. 


\section{Abstract}

$\alpha$-Synuclein inclusion bodies are a pathological hallmark of several neurodegenerative diseases. Although it has been hypothesized a relationship between fibril polymorphism and different pathologies, the molecular origins of polymorphism are not understood. Here, employing biophysical approaches, we demonstrate that the conformational state of the monomeric $\alpha$ Syn is responsible for fibril polymorphism: $\alpha$ Syn can exist as a compact monomer that produces rod fibrils, and as extended monomers that generate twisted fibrils. Using NMR, we found that the compaction relies on a polar interaction between the initial part of the NAC region and a wide section of the C-terminus domain. The compaction can be commonly affected by changes in the chemical environment, like $\mathrm{NaCl}$, the presence of $\mathrm{Ca}^{2+}$ or cellular components, like endotoxins, that alter the interaction NAC/C-terminus domain. Our compaction model also provides mechanistic insights that explain how the behavior of the $\mathrm{C}$-terminus domain imparts the polymorphism during the fibril formation. 


\section{Introduction}

The accumulation of alpha-synuclein ( $\alpha \mathrm{Syn})$ is a pathological hallmark of Parkinson's disease (PD) and multiple system atrophy (MSA) ${ }^{1-3}$; however, although these synucleinopathies are produced by the same protein, they exhibit clinically distinctive features. Recently, we have developed experimental strategies to study directly the structural characteristics of inclusions in patient's brain employing Fourier-Transform Infrared microscopy (FTIR) and Small-Angle X-Ray Scattering (SAXS), proving that Lewy Bodies (LBs) contain cross $\beta$-sheet $\alpha$ Syn fibrils, while the amount of $\beta$ sheet structure in LBs and glial cell inclusions in MSA patients is different ${ }^{4-6}$. These studies indicate that $\alpha$ Syn aggregates associated with PD and MSA correspond to different structural strains. Supporting our data, evidence corroborating distinctive strains in PD and MSA was provided analyzing amplified fibrils from the patients' brain using the protein misfolded cyclic amplification (PMCA) method ${ }^{7,8}$, and through the direct observation of accumulated fibrils in patient's brain by cryo-electron microscopy (Cryo-EM) ${ }^{9}$. Moreover, brain homogenates extracted from PD and MSA patients, as well as structurally different preformed fibrils, induced different cellular transmissibility, toxicity, membrane disruption, etc. ${ }^{710-16}$, leading to the hypothesis that pathological differences among synucleinopathies can be explained in terms of morphological variations in the accumulated $\alpha$ Syn fibrils in the brain ${ }^{17,18}$. In vitro, different strains of $\alpha$ Syn fibrils can be produced when the fibrils are generated under conditions of low or high ionic strength, whose characteristics resemble the morphological properties of the fibrils observed in PD and MSA patients ${ }^{16,19,20}$.

Despite the substantial progress in the analysis of strains amplified from patients' tissues and created in vitro, the molecular mechanisms that explain the origins of different strains from the same wild type $\alpha$ Syn remain poorly understood. To address this question, we focused on the conformational change of the monomeric $\alpha$ Syn. Several studies have reached a consensus about the disordered nature of $\alpha \mathrm{Syn}^{21-24}$; however, $\alpha$ Syn can also adopt some partially folded structures under acidic $\mathrm{pH}$ and high temperature ${ }^{25,26}$. Furthermore, some groups have proposed that these conformations represent intermediate species during the formation of fibrils ${ }^{27-29}$. Nevertheless, it remains unclear whether these conformational variations exist in the physiological environment and what their relationship is to polymorphisms. In this study, we propose that the molecular mechanisms responsible for polymorphisms are linked to the monomeric conformational state, which could be altered in the cell by the environment, eventually leading to the development of specific synucleinopathies. 


\section{Results}

\section{$\alpha$ Syn distinct fibril strains related to synucleinopathies can be modulated by the conformational state of the monomer}

To understand the morphological and biochemical characteristics of PD- and MSA-related fibril polymorphisms, we amplified amyloid fibrils from PD and MSA patients' brain by seeding, using amygdalae homogenates. The presence of $\alpha$ Syn aggregates in the amygdalae for all the cases was confirmed by immunohistochemical analysis (Fig. S1). First, we monitored the kinetics of fibril amplification by our HANABI assay ${ }^{30,31}$ (Figure 1.A), an instrument that consists of an optimized sonoreactor equipment developed by our group, that applies ultrasonic irradiation pulses individually to each one of the wells of a 96-wells microplate, and records the ThT fluorescence in function of time, named HANABI-2000 31 . This instrument accelerates substantially the rate of fibril formation reaction compared with the traditional RT-QuIC methods ${ }^{32,33}$, from several days to a few hours. Then, we analyzed the morphological properties by transmission electron microscopy (TEM, Figure 1.B). As previously reported ${ }^{8}$, we observed that fibrils amplified from PD patients' brain exhibit a higher ThT intensity than those obtained from MSA. The microscopic visualization showed that PD-amplified fibrils are characterized by rod-like fibrils, with two parallelly aligned protofilaments; while MSAderived fibrils depicted a twisted morphology, in which two protofilaments entangle over each other. These results highlight the importance of a deeper investigation to reveal the molecular mechanisms responsible for polymorphism.

Previous reports have shown that different fibril morphologies, each one with distinctive biophysical and biochemical properties, can be generated in vitro employing low or high ionic strength conditions ${ }^{16,19,20}$. A deeper knowledge of the fibril morphology phase diagram in function of the concentration of $\mathrm{NaCl}$ would enable us to understand the upstream molecular events related to the polymorphism of fibrils. Therefore, we examined the $\alpha$ Syn fibril formation by ThT assay in the presence of $50 \mathrm{mM}$ TRIS pH 7.4 supplemented with 0 to $500 \mathrm{mM} \mathrm{NaCl}$ (Figure 1.C). Interestingly, below $300 \mathrm{mM} \mathrm{NaCl}$, we found typical sigmoidal kinetic curves with relatively low maximal ThT intensity values (<0.2 A. U.). At $300 \mathrm{mM} \mathrm{NaCl}$, a wider distribution of curves with both low and high maximal ThT was detected, while above $400 \mathrm{mM} \mathrm{NaCl}$, just fibrils with high values of ThT intensity were observed. To determine the phase diagram of ThT intensity of fibrils, we plotted the maximal values of $\mathrm{ThT}$ as a function of the concentration of $\mathrm{NaCl}$, finding a region dominated by low maximal ThT values below $300 \mathrm{mM} \mathrm{NaCl}$, and a region with high maximal ThT values above $300 \mathrm{mM}$. We defined the inflection point of the phase diagram as the concentration of $\mathrm{NaCl}, 300 \mathrm{mM}$, in which fibrils with both low and high ThT can be produced by chance. Moreover, we evaluated by TEM the morphological properties of the fibrils created at different concentrations of $\mathrm{NaCl}$ (Figure 1.D, upper and lower-right). First, it was found that the low-ThT fibrils obtained below $300 \mathrm{mM} \mathrm{NaCl}$ exhibit a 
twisted morphology, with similar characteristics than those observed in the MSA-amplified fibrils. The fibrils produced above $300 \mathrm{mM} \mathrm{NaCl}$ with high-ThT exhibit a rod morphology, resembling the properties of the PD-derived fibrils.

In addition, we also confirmed by SDS-PAGE densitometry that the yield of both the low and high ThT fibrils created at low and high salt, respectively, is higher than $95 \%$ (Fig. S2.A), discarding the possibility of incomplete reactions or the lack of fibrillation products when low ThT is observed. Finally, we recorded the FTIR spectra of both low-salt and high-salt fibrils, finding in both cases typical bands of $\beta$-sheet structure around $1630 \mathrm{~cm}^{-1}$, demonstrating the presence of amyloid fibrils in both $\mathrm{NaCl}$ conditions, and excluding the formation of amorphous aggregates (Fig. S2.B). The deconvolution of the FTIR spectra showed that the contents of $\beta$-sheet structure is higher for the fibrils created at $50 \mathrm{mM} \mathrm{NaCl}$, indicating structural differences between the fibrils (Fig. S2.C).

For the biochemical characterization of fibrils, we developed a proteinase $\mathrm{K}$ resistance assay (PKR) to evaluate and compare the digestion patterns of fibrils with different morphologies. Considering the first five bands with the highest molecular in the proteolysis patterns after 60 minutes of reaction, rod-like fibrils showed a digestion profile with stronger band intensities for the second (B2) and fourth (B4) bands, while twisted fibrils presented a weaker B2 band (Fig. S2.D-E). Employing this PKR assay, we evaluated the digestion patterns of fibrils obtained at $50 \mathrm{mM}$ and 500 $\mathrm{mM} \mathrm{NaCl}$ concentrations, where the differential digestion profiles for twisted and rod fibrils, respectively, were confirmed (Figure 1.E, left). Furthermore, we tested the PKR assay of selected fibrils created at $300 \mathrm{mM} \mathrm{NaCl}$ with low and high maximal ThT fibrils, whose digestion patterns are in good agreement with the morphology visualized by TEM (Figure 1.E, center). Finally, we also evaluated the digestion patterns of fibrils amplified from PD and MSA brain samples, in which degradation profiles of rod-like and twisted morphologies, respectively, were detected (Figure 1.E, right), corroborating the biochemical similarities between in vitro created fibrils and fibril derived from patients' brain. Taken together, these results corroborated that the transition of maximal ThT values in function of the concentration of $\mathrm{NaCl}$ reflects the morphological phase diagram of the fibrils, which might explain the origins of the fibril diversities in the patients of PD and MSA.

We then explored the upstream molecular events that influence the phase diagram of the fibrils. It has been reported previously that $\alpha$ Syn can adopt different conformational states in solution ${ }^{21,23,26}$. In order to investigate whether these conformations are related to the molecular events in the early monomeric state that lead to the formation of specific fibrils, we started solving a solution of $\alpha$ Syn by analytical size exclusion chromatography (SEC) at $300 \mathrm{mM} \mathrm{NaCl}$ (Figure 1.F, upper). At this ionic strength, $\alpha$ Syn can produce both rod and twisted morphologies. Interestingly, the full-length $\alpha$ Syn monomer, whose molecular weight is $14.6 \mathrm{kDa}$, was observed as a single asymmetric peak, widely 
distributed in retention volume values ranging from molecular weights of $15 \mathrm{kDa}$ to $60 \mathrm{kDa}$ for hypothetical spherical-shaped globular proteins. The peak center of $\alpha$ Syn was observed around the retention volume that corresponds to $50 \mathrm{kDa}$ globular proteins. This result suggests that $\alpha \mathrm{Syn}$ monomers can adopt conformations from compact to more extended states in which the apparent hydrodynamic size increases. We confirmed by Western blotting that all the collected $\alpha$ Syn fractions separated by SEC consist of intact full-length monomers that have access to conformations with different hydrodynamic radii (Figure 1.F, lower). In addition, we also evaluated the retention volume of the monomeric $\alpha$ Syn between 50 and $500 \mathrm{mM} \mathrm{NaCl}$, finding a constant increase in the retention volume between $50 \mathrm{mM}$ and $300 \mathrm{mM} \mathrm{NaCl}$, but no significant change above $300 \mathrm{mM} \mathrm{NaCl}$ (Figure 1.G and Fig. S3.A), indicating that the monomers have reached a plateau conformational phase around $300 \mathrm{mM} \mathrm{NaCl}$, in which the protein is found in a more compact conformation than in lower concentrations of $\mathrm{NaCl}$. Moreover, we evaluated the retention volume of solutions of bovine serum albumin (BSA), in the presence of $\mathrm{NaCl}$ at $150 \mathrm{mM}$ and $500 \mathrm{mM}$ (Fig. S3.B, C). No variation in the retention volume was detected, corroborating that the changes in the retention volume observed in $\alpha$ Syn are not an artifact of the differences in the ionic strength.

A more exhaustive evaluation of the hydrodynamic radius of the monomeric $\alpha$ Syn was performed using Analytical Ultracentrifugation (AUC) at low (150 mM) and high (500 $\mathrm{mM})$ concentrations of $\mathrm{NaCl}$. By AUC Sedimentation Velocity, we observed a significant decrease in the values of the sedimentation coefficient, from $1.136 \pm 0.012 \mathrm{~S}$ at $150 \mathrm{mM} \mathrm{NaCl}$ to $1.108 \pm 0.011 \mathrm{~S}$ at $500 \mathrm{mM} \mathrm{NaCl}$ (Figure 1.H), indicating a compaction from $150 \mathrm{mM}$ to $500 \mathrm{mM} \mathrm{NaCl}$. These measurements also confirmed that $\alpha$ Syn is found mainly as a monomer $(>98 \%)$. Taken together, our results indicate that the size distribution of the monomeric $\alpha$ Syn changes from an extended conformation at low ionic strength to a more compact form above $500 \mathrm{mM} \mathrm{NaCl}$.

\section{The behavior of the C-terminus determines the compaction of $\alpha$ Syn}

To determine the molecular events responsible for the compaction of $\alpha$ Syn monomers, we employed different $\mathrm{NaCl}$ concentrations $(0-500 \mathrm{mM})$ to record ${ }^{1} \mathrm{H}-{ }^{15} \mathrm{~N}$ HSQC spectra (Figure 2.A and Fig S4.A). The analysis of the peak intensities as a function of $\mathrm{NaCl}$ concentration, using the spectrum obtained at $0 \mathrm{mM} \mathrm{NaCl}$ as reference, allowed us to detect a constant decrease in the peak intensity at the initial part of the NAC region and in the majority of residues in the C-terminal domain residues (Figure 2.B and Fig. S4.B, left), detected by both difference $\left(I_{\mathrm{x}}-I_{0 \mathrm{mM}}\right)$ and ratio $\left(I_{\mathrm{x}} / I_{0 \mathrm{mM}}\right)$ of peak intensities. Additionally, some peaks disappeared upon the increase in $\mathrm{NaCl}$ concentration, located mostly in the NAC region (Fig. S4.B, marked with *). Remarkably, the peaks that experienced the most significant changes in the NAC domain were attributed to aliphatic and non-charged polar residues. Among the peaks showing changes in the $\mathrm{C}$-terminal domain, half were attributed to 
aliphatic, aromatic, or non-charged polar residues, and the others to negatively charged residues (Figure 2.C). The analysis of chemical shift perturbation (Fig. S2.B, right) did not show significant variations.

To determine the relative flexibility differences between the monomers at distinct ionic strength, we performed heteronuclear Overhauser effect (hetNOE) experiments with $\alpha$ Syn incubated in $50 \mathrm{mM}$ TRIS pH 7.4 supplemented with $0 \mathrm{mM}$ and $500 \mathrm{mM} \mathrm{NaCl}$ (Fig. S4.C). We found that most of the changes, but not the only ones, were seen in the NAC region (highlighted with a gray square), where several residues show more negative hetNOE values at $0 \mathrm{mM} \mathrm{NaCl}$, while at $500 \mathrm{mM} \mathrm{NaCl}$ some of these residues exhibit a higher hetNOE values or they have even disappeared in ${ }^{1} \mathrm{H}-{ }^{15} \mathrm{~N}$ HSQC spectrum with ${ }^{1} \mathrm{H}$ saturation. The $\mathrm{C}$-terminal domain shows just few changes between the residues 120 and 130, where hetNOE is higher. These results suggest that although the monomers retain their intrinsically disordered nature (Fig. S4.D), at higher ionic strength, both the NAC region and the Cterminal domain suffer a decrease in the flexibility, probably as consequence of the compaction of the monomer.

Additionally, we explored 576 structures deposited in the Protein Ensemble Data Bank, PED $00024^{34}$ to corroborate the experimental HSQC spectra. In all the salt concentrations, the closest structure to our data, evaluated by the lowest root-mean-square (RMS), was an extended conformation (Fig. S4.E, left). Interestingly, the second lowest RMS value at $500 \mathrm{mM} \mathrm{NaCl}$ had a more compact conformation with the C-terminal domain interacts with the central region of the protein (Fig. S4.E, right), and the RMS value of this structure decreased as the salt concentration increased. These results are consistent with our experimental data that the formation of compact monomer increased when $\mathrm{NaCl}$ was added.

\section{Calcium ions modulate the monomeric compaction and shift the inflection point of fibril} morphologies to the rod fibril.

It has been reported that the cation $\mathrm{Ca}^{2+}$ binds to the negatively charged residues of the $\mathrm{C}$ terminal domain, and alters the conformation of $\alpha \mathrm{Syn}^{35}$. With this context, we assessed $\mathrm{Ca}^{2+}$ ions as modulators of the conformational state of $\alpha$ Syn. First, we examined the fibril formation in presence of $200 \mathrm{Eq}$. $\mathrm{Ca}^{2+}$ at different ionic strength, ranging from 21 to $500 \mathrm{mM}$ (Figure 3.A and Fig S5.A). In the presence of $\mathrm{Ca}^{2+}$, below $200 \mathrm{mM}$ ionic strength (IS) a variety of ThT values was seen, while above $200 \mathrm{mM}$ IS just high ThT fibrils were observed. These results indicate that calcium promotes the formation of fibrils with high ThT at lower IS, and even prevents the formation of exclusively low ThT fibrils at low IS. The morphology of the fibrils formed at an IS of $200 \mathrm{mM}$ in the presence of $\mathrm{Ca}^{2+}$ was tested by TEM (Figure 3.B) and PKR (Figure 3.C), that confirmed the morphological properties of the rod fibrils. In order to verify the relationship between fibril morphology and monomeric 
conformation, we solved solutions of $\alpha \mathrm{Syn}$ in the presence and absence of $200 \mathrm{Eq} \cdot \mathrm{Ca}^{2+}$ at an IS of $200 \mathrm{mM}$ by SEC (Figure 3.D), finding a significant higher retention volume when calcium is present in the solution and suggesting a connection between the rod morphology and a compact monomeric state.

To determine the residues affected in the compact monomer induced by calcium, we recorded ${ }^{1} \mathrm{H}-{ }^{15} \mathrm{~N}$ HSQC spectra in the presence and absence of $200 \mathrm{Eq} . \mathrm{Ca}^{2+}$ at an ionic strength equivalent to $200 \mathrm{mM} \mathrm{NaCl}$ (Figure 3.E). The analysis of the peak intensities by both difference $\left(I_{200 \mathrm{Ca}}-I_{0 \mathrm{Ca}}\right)$ and ratio $\left(I_{200 \mathrm{Ca}} / I_{0 \mathrm{Ca}}\right.$ ), using the spectrum recorded in the absence of $\mathrm{Ca}^{2+}$ at $200 \mathrm{mM} \mathrm{NaCl}$ as reference, showed again a decrease in the peak intensity at the initial part of the NAC region and in residues in the C-terminal domain residues (Figure 3.F, upper and middle). The peaks showing changes share similarities with those detected by $\mathrm{NaCl}$ dependence in both the NAC region and in the C-terminus domain. The analysis of chemical shift perturbation (Figure 3.F, lower) did not show significant variations. These results indicate that the compaction achieved by high ionic strength are also involved when the compaction is enhanced by calcium. A similar analysis employing the spectrum recorded at $0 \mathrm{mM}$ IS as reference indicate more intense variations in the peak intensity values (Fig. S5.B, C), however this comparison cannot distinguish the contribution attributed to the ionic strength from the effect of calcium.

To determine the relative flexibility in the presence and absence of $\mathrm{Ca}^{2+}$ at $200 \mathrm{mM}$ IS, we performed hetNOE (Fig. S5.D). The detected changes were observed at the initial part of the NAC region, where in the presence of $\mathrm{Ca}^{2+}$ several residues show less negative hetNOE values or zero. The C-terminal domain shows slight changes around the residue 110, and more clearly around the residue 120 , where hetNOE value is higher. These results, in a similar way to the situation at higher ionic strength, confirm that both the NAC region and the C-terminal domain suffer a decrease in the flexibility, as consequence of the compaction of the monomer.

\section{The cellular environment alters the conformational state of $\alpha$ Syn}

It has been reported that different cellular environments and their components can promote the formation of specific polymorphs ${ }^{12}$. One interesting case is observed during the bacterial dysregulation of the microbiome in the gut, phenomenon that has been related to the development of PD. The release of microbial membrane metabolites, like Lipopolysaccharides (LPS), could represent a crucial mediator of $\alpha$ Syn amyloidogenesis via the gut-brain axis ${ }^{36}$. To investigate the effect of the bacterial cellular components in the conformational state of the monomeric $\alpha$ Syn, and fibril morphology, we designed an alternative purification protocol in which some bacterial elements remained in the protein preparation, namely IEX- $\alpha$ Syn. Our standard purification strategy of $\alpha$ Syn (see Materials and Methods section) includes SEC and reverse phase chromatography after ion exchange chromatography 
(IEX) as final steps to completely remove molecules with similar charge than $\alpha$ Syn. From this point, we will call this protein HPLC- $\alpha$ Syn. However, IEX- $\alpha$ Syn preparation lacks the final steps of purification in order to keep in the protein mixture some components of the bacterial environment. Then, we analyzed and compared the chromatograms of both standard $\alpha$ Syn (HPLC- $\alpha$ Syn) and IEX$\alpha$ Syn by analytical SEC (Figure 4.A) and analytical reverse phase chromatography (Figure 4.B), showing the presence of a component with small size (dashed circle) but constituted by elements with different hydrophobicity (black arrows). The peaks detected by reverse phase chromatography were grouped by retention time, those with a shorter and those with a longer retention time than the peak of $\alpha$ Syn, analyzed by electrospray ionization mass spectrometry (ESI-MS) and deconvoluted to estimate the mass of the molecular ion (Fig. S6.A). We found, for both groups, that the molecular weight of the bacterial components was $\sim 12-14 \mathrm{kDa}$. Furthermore, employing the low molecular weight fraction marked in Figure 4.A, we recorded its FTIR spectrum (Fig. S6.B), finding two bands between 1700 and $1500 \mathrm{~cm}^{-1}$. The molecular weight determined by ESI-MS is in good agreement with the value expected for bacterial endotoxins, that range between 10 and $20 \mathrm{kDa}^{37}$. The bands detected by FTIR could correspond to the Amide I and Amide II of LPS ${ }^{38}$, while the heterogeneity in the number of carbohydrate moieties in LPS could explain the different hydrophobicity peaks observed by analytical reverse phase chromatography. Finally, to corroborate the presence of LPS in IEX- $\alpha$ Syn, we employed a Toxin Sensor Kit (GenScript), finding an LPS-reactive result (Fig. S6.C). Taken together, these results clearly show that most of the bacterial elements in IEX- $\alpha$ Syn corresponds to LPS.

In order to understand the effect of the bacterial elements in the fibril morphology, we monitored the formation of IEX- $\alpha$ Syn fibrils by ThT assay in $50 \mathrm{mM}$ TRIS pH 7.4 supplemented with $\mathrm{NaCl}$ ranging from 0 to $500 \mathrm{mM}$ and compared with the morphology profile obtained in the Figure 1.D for the HPLC- $\alpha$ Syn (Figure 4.C). The results indicate that the bacterial components induce a ThT inflection point at a lower ionic strength $(\sim 150 \mathrm{mM})$ than the observed for HPLC- $\alpha$ Syn $(\sim 300 \mathrm{mM})$. The morphological consequences become evident at $200 \mathrm{mM} \mathrm{NaCl}$, conditions in which IEX- $\alpha$ Syn forms a fibril with higher ThT that exhibits a typical rod morphology (Figure 4.C, red square), while pure $\alpha$ Syn produces a fibril with lower ThT and twisted polymorph (Figure 4.C, gray square). Since the LPS molecule was identified as the main bacterial component in IEX- $\alpha$ Syn, we prepared an artificial IEX- $\alpha$ Syn by adding LPS to HPLC- $\alpha$ Syn and tested the fibril morphology phase diagram by ThT assay (Figure 4.D), finding a ThT inflection point of $\sim 150 \mathrm{mM}$, in good agreement with the value determined for IEX- $\alpha$ Syn. The morphological differences produced by the change in the inflection point of morphologies were also corroborated by TEM at $300 \mathrm{mM} \mathrm{NaCl}$, ionic strength in which LPS added $\alpha$ Syn shows a rod morphology and the HPLC- $\alpha$ Syn a twisted fibril (Figure 4.D, purple and black squares, respectively). 
Next, we performed ${ }^{1} \mathrm{H}^{-15} \mathrm{~N}$ HSQC experiments to determine the residues affected by the presence of bacterial endotoxins in IEX- $\alpha$ Syn and compared with the spectrum of HPLC- $\alpha$ Syn, at a concentration of $\mathrm{NaCl}$ of $200 \mathrm{mM}$, in which the differences between IEX- $\alpha$ Syn and HPLC- $\alpha$ Syn are clearer (Figure 4.E). The peak intensities difference ( $I_{\text {IEX }}-I_{\text {HPLC }}$ ) shows a slight variation in all the three domains of $\alpha$ Syn, while the ratio $\left(I_{\mathrm{IEX}} / I_{\mathrm{HPLC}}\right)$ indicates that the most intense changes are located in the N-terminus domain and the NAC region (Figure 4.F), while the C-terminus domain exhibits decreases in the values of ratio at the initial part of the C-terminus region and around the residue 130 . The chemical shift perturbation shows no significant differences. Interestingly, using the artificial IEX- $\alpha$ Syn (HPLC- $\alpha$ Syn + LPS), its ${ }^{1} \mathrm{H}^{-15} \mathrm{~N}$ HSQC spectrum is very similar to that obtained for IEX$\alpha$ Syn (Figure 4.G). The similarities are clearer if we consider the peak intensities difference ( $I_{\text {HPLC+LPS }}$ - $\left.I_{\text {HPLC }}\right)$ and ratio ( $I_{\text {HPLC+LPS }} / I_{\text {HPLC }}$, where the regions mostly affected by the presence of LPS are almost identical to those observed in IEX- $\alpha$ Syn (Figure 4.H). No significant differences were seen by chemical shift perturbation. These results suggest that the $\mathrm{N}$-terminus domain and some residues in the NAC region are responsible for the interaction with the bacterial endotoxins. However, in order to determine if there is induced by bacterial endotoxins, we established an algorithm of peak intensities based on the compact state observed at $300 \mathrm{mM} \mathrm{NaCl}$ (Fig. S7.A). First, we defined the total variation in peak intensity ( $v_{\text {total }}$ ) of a residue $(r)$ by subtracting from the highest peak intensity, found at $0 \mathrm{mM}$ $\mathrm{NaCl}\left(I^{0 \mathrm{mM}}\right)$, the lowest peak intensity, at $500 \mathrm{mM} \mathrm{NaCl}\left(I^{500 \mathrm{mM}}\right)$ :

$$
v_{\text {total }}(r)=I^{0 m M}(r)-I^{500 m M}(r)
$$

We also defined the variation of a peak intensity, at a certain ionic strength ([I.S.]), affected by the X agent, like $\mathrm{Ca}^{2+}$ or LPS, as:

$$
v_{X}^{[I . S .]}(r)=I^{0 m M}(r)-I_{X}^{[I . S .]}(r)
$$

Then, we normalized the variation of a peak intensity using the total variation of peak intensity:

$$
c_{X}^{[I . S .]}(r)=\frac{v_{X}^{[I . S .]}(r)}{v_{\text {total }}(r)}
$$

Since above $300 \mathrm{mM} \mathrm{NaCl}$ we detected a plateau phase of compaction by SEC, as shown in Figure 1.G, we defined the threshold of compaction for the intensity of a peak as its $c(r)$ value at 300 $\mathrm{mM} \mathrm{NaCl}$ :

$$
c^{300 m M}(r)=\frac{v^{300 m M}(r)}{v_{\text {total }}(r)}
$$

Thus, those residues in the condition $\mathrm{X}$ that can induce a normalized variation in their peak intensities higher than their $c^{300 \mathrm{mM}}(r)$, have been considered as relevant for compaction (marked as the red area in Fig. S7.A): 


$$
\frac{c_{X}^{[I . S .]}(r)}{c^{300 m M}(r)} \geq 1.0
$$

Additional constraints were introduced. For instance, those residues whose $v_{\text {total }}$ is lower than 0.1 and their normalized ratio of intensities $\left(I^{[\mathrm{IIS} .} \mathrm{X} / I^{300 \mathrm{mM}}\right)$ is higher than 0.75 have been omitted to avoid false positives.

Using the algorithm described above, we performed a global analysis of the peak intensities at an ionic strength equivalent to $200 \mathrm{mM} \mathrm{NaCl}$ in the presence of bacterial endotoxins (IEX- $\alpha$ Syn), LPS (HPLC- $\alpha$ Syn + LPS), and $\mathrm{Ca}^{2+}$ (Fig. S7.C). This analysis allowed us to recognize common residues affected under the tested conditions. For instance, while the N-terminus domain showed as common disturbed residue just the A11, the NAC region exhibits clearer variations at its initial part, specifically the residues Q62 and N65. The most affected region for all the conditions was the C-terminus domain, in which several residues between G106 and E139 surpassed the threshold established by $c^{300 \mathrm{mM}}(r)$ (Fig. S7.B), suggesting that the bacterial endotoxins induce a similar conformational compaction when interacting with $\alpha$ Syn than the observed at high ionic strength or in the presence of calcium. An alignment comparing the most relevant changes detected by the defined algorithm applied to all the conditions of high $\mathrm{NaCl}, \mathrm{Ca}^{2+}$ at $200 \mathrm{mM}$ I.S., bacterial endotoxins in IEX- $\alpha$ Syn at $200 \mathrm{mM}$ I.S., and LPS-added to HPLC- $\alpha$ Syn at $200 \mathrm{mM}$ I.S., is provided in Figure 4.I. The global analysis shows two main affected regions for all the cases: the initial part of the NAC region and a wide area of the Cterminal domain. Considering these results, it is clear that all the tested conditions share a common molecular mechanism, in which there is a compaction enhanced by the interaction between the initial part of the NAC region and the $\mathrm{C}$-terminus domain that precedes the formation of rod fibrils.

\section{The C-terminus domain is essential for the monomeric compaction and fibril polymorphism.}

We have identified the C-terminus domain as a key factor in the compaction of the monomeric $\alpha$ Syn. If we examine the sequence of the $\mathrm{C}$-terminus domain, we will find four aromatic residues: one phenylalanine and three tyrosine residues. Among the tyrosine residues, two of them were directly identified by the global algorithm employed to analyze the NMR data (Y133 and Y136), suggesting that the chemical environment of the tyrosine residues could change upon the compaction of $\alpha$ Syn (Fig. S8.A). Since the aromatic residues can be studied relatively easily with spectroscopic tools, we employed near circular dichroism (CD) spectroscopy to evaluate the alterations in the tyrosine environment in the presence of components that induce the compaction of monomers, like high concentration of $\mathrm{NaCl}$ and calcium (Fig. S8.B). The spectra showed some differences in the region of the tyrosine residues $(265-285 \mathrm{~nm})$, whose ellipticity values become less negative in conditions that promote the compaction, while the phenylalanine residues region $(255-265 \mathrm{~nm})$ remained unaltered. 
These results indicate that spectroscopic tools that target the aromatic residues could be implemented as first approaches to detect the compact conformation in $\alpha$ Syn.

Finally, since a wide section of the C-terminus domain has been recognized as crucial for the compaction of the monomeric $\alpha$ Syn, we examined a truncated form of $\alpha$ Syn that lacks this region, from E105 to A140, namely N103t- $\alpha$ Syn, (Figure 5.A). First, we monitored the fibril formation using N103t- $\alpha$ Syn at different concentrations of $\mathrm{NaCl}$, ranging from $50 \mathrm{mM}$ to $300 \mathrm{mM}$, finding that, independently from the ionic strength, the ThT values for all the fibrils were low (Figure 5.B). A TEM visualization of the fibrils showed that just twisted fibrils were detected, even at high concentrations of salt (Figure 5.C). Additionally, we evaluated if there were changes in retention volume by analytical SEC, however, the retention volume did not show any significant variation in the interval from $50 \mathrm{mM}$ to $500 \mathrm{mM} \mathrm{NaCl}$. These results indicate that the lack of the key region of the C-terminal domain is enough to prevent not just the formation of rod fibrils but also any variation in the conformational state of the monomeric N103t- $\alpha$ Syn.

\section{Discussion}

The direct visualization of fibril polymorphs extracted from the brain of DLB and MSA patients represent an unprecedent evidence for the hypothesis that different polymorphs are related to the development of different diseases ${ }^{9}$. In vitro, the methods to create a variety of $\alpha$ Syn fibril morphologies, altering conditions like the ionic strength, are well known ${ }^{7,19,20}$; however, the molecular mechanisms that generate those diverse polymorphs from the same intrinsically disordered protein remain poorly understood. In this study, employing physicochemical approaches, we have found that, at neutral $\mathrm{pH}$, the local environment of $\alpha$ Syn can induce a conformational compaction of the monomeric protein, which is related to the specific formation of polymorphs. Our results of SEC and AUC indicate that if the ionic strength increases, the protein suffers a compaction that reaches a stable level above $300 \mathrm{mM} \mathrm{NaCl}$, that corresponds with the region in the morphology phase diagram in which the formation of rod fibrils begins. The structural elements of the monomeric compaction, induced by $\mathrm{NaCl}$, were revealed by NMR, in which variations in the initial part of the NAC region, specifically the residues Q62 and N65, and a wide area of the C-terminus domain suggests the existence of a polar interaction between these two regions. The identification of the C-terminus domain as key factor for the compaction is particularly remarkable since this region is the target of several modifications, like phosphorylation, cross-linking, ubiquitination, truncation, interaction with metals, etc. ${ }^{39}$, events that could potentially affect the conformational state of $\alpha$ Syn and the consequent fibril polymorph.

Recently, it was reported, at low ionic strength, the interaction between $\alpha \mathrm{Syn}$ and $\mathrm{Ca}^{2+}$, a biologically relevant cation that also accelerates the speed of fibril formation ${ }^{35}$. The authors found that 
the interaction between the negative residues of the $\mathrm{C}$-terminal domain and $\mathrm{Ca}^{2+}$ promotes a disruption in the electrostatic contacts with the positively charged $\mathrm{N}$-terminus domain, leading to a higher exposure of the N-terminus domain and the initial part of the NAC region. Our results confirm the interaction between the C-terminus domain and $\mathrm{Ca}^{2+}$ (Figure 3), and also provide a more general mechanism based on the monomeric compaction applicable not only to the reported low ionic strength $^{35}$, but also for mild (physiological) and high ionic strength. First, in the presence of $200 \mathrm{Eq}$. $\mathrm{Ca}^{2+}$, we found that $\alpha$ Syn can form fibrils with both low and high ThT values when the ionic strength is low. However, above an ionic strength of $200 \mathrm{mM}$, just fibrils with rod morphology and high ThT were observed. Furthermore, at this ionic strength, we detected a compaction in the monomer (Fig 3.D) accompanied by structural modifications, detected by NMR, in the initial part of the NAC region and in a wide area of the $\mathrm{C}$-terminus domain, similarly to those residues observed at high concentration of $\mathrm{NaCl}$. Taken together, our results suggest that, independently of the ionic strength, $\mathrm{Ca}^{2+}$ establishes electrostatic interactions with the residues of the $\mathrm{C}$-terminus domain, titrating the negative charges of residues like Glu and Asp. However, the $\mathrm{Ca}^{2+}$-bound-C-terminus domain behaves differently when the ionic strength changes. At low ionic strength, the titrated C-terminus domain will get released from the transient electrostatic contacts with the $\mathrm{N}$-terminus region, however, the interaction with the initial part of the NAC region still might occur sporadically; in consequence, the fibrils can acquire both the rod and the twisted morphology. When the ionic strength reaches $200 \mathrm{mM}$, the concentration of ions in solution induces a tendency in $\alpha$ Syn to protect its hydrophobic NAC region from the solvent. Since $\mathrm{Ca}^{2+}$ has partially neutralized some negative charges of the $\mathrm{C}$-terminus domain, the interaction with the NAC region gets facilitated with the concomitant compaction of the monomer. In the absence of calcium, the negative charges of the $\mathrm{C}$-terminus domain need a higher ionic strength, like $300 \mathrm{mM}$, to get neutralized and allow the interaction with the initial part of the NAC region. Thus, calcium enhances the compaction of the monomer and promotes the formation of rod fibrils at lower ionic strength values than $\mathrm{NaCl}$, in the range of the physiological ionic strength. The compaction of monomers induced by calcium could trigger the fibril accumulation of rod fibrils during the dysregulation of intracellular calcium homeostasis, event that has been found to play an important role in the pathogenesis of $\mathrm{PD}^{40}$.

The effect of some components present in the cellular environment on the compaction of monomers was evaluated employing bacterial endotoxins (LPS), that are molecules associated to the $\alpha$ Syn amyloidogenesis through the gut-brain axis $^{36}$. The ThT assay evaluation of fibril morphologies indicated the formation of rod fibrils at lower ionic strength than salt. For example, at an ionic strength of $200 \mathrm{mM}$, pure $\alpha$ Syn shows twisted fibrils while IEX- $\alpha$ Syn just shows rod fibrils. This decrease in the ThT inflection point of fibril morphologies suggests that an enhanced compaction of monomers at lower ionic strength could take place in the presence of bacterial endotoxins. The NMR measurements 
indicate that the binding between LPS and $\alpha$ Syn occurs involving the $\mathrm{N}$-terminus domain and some hydrophobic residues of the NAC region. Interestingly, The driving force of the interaction could be a series of hydrogen bonds established between the positively charged $\mathrm{N}$-terminus domain and the hydroxyl groups in the carbohydrate moieties of LPS, while hydrophobic contacts might take place between the lipidic aliphatic chains of LPS and the hydrophobic NAC region. However, our algorithm could recognize affected regions in the initial part of the NAC region and a wide area of the $\mathrm{C}$-terminus domain, that are in good agreement with the residues identified at high ionic strength and in the presence of $\mathrm{Ca}^{2+}$, and implying that the phenomenon of compaction also happens in the presence of endotoxins. These results suggest a mechanism of compaction: when LPS binds the N-terminus domain and some residues of the NAC region, the C-terminus domain gets released from the electrostatic interaction with the N-terminus domain and, since the hydrophobic residues of the NAC region are compromised with the aliphatic region of LPS, the polar interaction between the residues Q62 and N65 with the C-terminus domain gets facilitated, leading to the formation of a compact monomer bound to LPS, which is also able to produce rod fibrils at lower ionic strength. These results have a critical importance since they provide a new mechanism of fibril formation when the microbiota in the gut is dysregulated, and the bacterial endotoxins concentration increases. In addition, our findings also suggest that the binding of $\alpha$ Syn to other cellular components through the $\mathrm{N}$-terminus domain, like the cellular membranes, could induce not only the acceleration of fibril aggregation ${ }^{41}$, but also might expose the NAC region to the interaction with the C-terminus domain and lead to the monomeric compaction and the subsequent formation of specific polymorphs.

The conditions examined here (differences in ionic strength, the presence of calcium, the effect of bacterial endotoxins) modified the phase diagram of fibrils by affecting the monomeric conformation of $\alpha$ Syn and the interaction between the C-terminus domain with the initial part of the NAC region. In physiological conditions of $\mathrm{pH}$ and ionic strength, our morphological phase diagram indicates that the preferred fibril would be the twisted polymorph; however, the dysregulation of metabolites like LPS and calcium, or, hypothetically, lipids like glucosylceramide ${ }^{42}$, might alter this phase diagram and enable the aggregation of rod fibrils, like those observed in PD. The model of the monomeric compaction and the phase diagram of fibrils could be expanded to the complexity of the cellular environments. For instance, Peng et $a l^{12}$ reported that the $\alpha$ Syn aggregates in the neuronal cells can produce PD-like aggregates, whereas the $\alpha$ Syn aggregated in the oligodendrocyte resembles the MSA-like ones. Further investigation is required to determine the fibril phase diagrams in the neuronal cells and in the oligodendrocytic cells and the factors that trigger the aggregation in these environments. This knowledge would provide insights to better understand the pathogenesis of each alpha synucleinopathies. 
Finally, although our model of compaction allows the prediction of fibril morphologies, the direct link explaining how the monomeric compaction and fibril morphology are related remains unsolved. Considering our results and the cryo-EM polymorphs structures already reported ${ }^{43,44}$, we propose a mechanism for the formation of both twisted and rod fibrils from the scenario of the monomeric compaction: 1) (Figure 6, left) at low ionic strength, the monomer adopts an ensemble of extended conformations with structural fluctuations. During the fibril formation, the structural monomeric plasticity allows the protein to acquire the most stable morphology: the twisted fibril. The driving force of the twisted fibril would be the protection from the solvent of the hydrophobic residues of the NAC region at the interface of the constituting protofilaments. 2) (Figure 6, right) Under conditions that promote the compaction of monomers, like high ionic strength, calcium or LPS, the Cterminus domain that interacts with the initial part of the NAC region would protect the hydrophobic residues of this domain, preventing the hydrophobic contact between the protofilament interfaces required for the twisted fibril. Instead, an interface characterized by the electrostatic interaction between H50 and E57 and a hydrophobic zipper from G51 to A56 would be stabilized, leading to the formation of the rod fibril.

\section{Materials and Methods}

All chemicals and reactants were obtained from Sigma-Aldrich or Nacalai Tesque (Kyoto, Japan). All reagents were used without further purification. Water was purified to a resistivity of $18{\mathrm{M} \Omega \mathrm{cm}^{-1}}^{-1}$ using a Millipore Gradient deionizing system.

\section{$\underline{\text { Protein purification }}$}

Human WT $\alpha$ Syn was purified from Escherichia coli as described previously (34). Briefly, a plasmid containing WT human aSyn was expressed in E. coli BL21 (DE3) cells (Novagen, Merck, San Diego, CA, USA). The cells were suspended in purification buffer, disrupted by sonication, and centrifuged. Streptomycin sulfate (final $2.5 \% \mathrm{w} / \mathrm{w}$ ) was added to the supernatant, and centrifugation was repeated. The supernatant was heated to $85^{\circ} \mathrm{C}$ in a water bath and centrifuged. The supernatant was precipitated by the addition of solid ammonium sulfate to $70 \%$ saturation, centrifuged, dialyzed overnight, applied to a Resource-Q column (GE Healthcare, Little Chalfont, UK) with $50 \mathrm{mM}$ TRIS-HCl pH7.4 buffer containing $0.1 \mathrm{mM}$ dithiothreitol and $0.1 \mathrm{mM}$ phenylmethylsulfonyl fluoride as a running buffer, and eluted with a linear gradient of $0-1 \mathrm{M} \mathrm{NaCl}$. $\alpha$ Syn-enriched fractions (as determined by sodium dodecyl sulfate-polyacrylamide gel electrophoresis SDS-PAGE/Coomassie blue staining) were pooled and further purified by size exclusion chromatography (SEC) using a Superdex 200 26/600 PG column (GE Healthcare, Little Chalfont, UK) equilibrated with $50 \mathrm{mM}$ TRIS-HCl pH 7.4 supplemented with $150 \mathrm{mM} \mathrm{NaCl}$. The fractions containing $\alpha$ Syn (as determined by SDS-PAGE/Coomasie blue staining) were joint, dialyzed versus deionized water, acidified with $5 \mathrm{mM} \mathrm{HCl}$ and loaded onto a Reverse Phase 
Cosmosil Protein R x 250 mm Preparative Column (Nacalai-Tesque, Kyoto, Japan) and eluted with a linear gradient of $30 \%-90 \%$ acetonitrile. The pure fractions were combined and flash-frozen in liquid nitrogen, lyophilized and stored at $-80{ }^{\circ} \mathrm{C}$ until use. The protein purity was confirmed to be greater than $95 \%$ by SDS-PAGE and matrix-assisted laser desorption/ionization mass spectrometry. The protein concentration was determined by UV absorption at $280 \mathrm{~nm}$ using an extinction coefficient $\varepsilon 1 \%$ of $3.54{ }^{45}$.

C-terminal truncated N103t $\alpha$ Syn: The C-terminal truncated $\alpha$ Syn construct was prepared using a QuikChange site-directed mutagenesis kit (Stratagene, Santa Clara, California), employing the WT$\alpha$ Syn plasmid of the previous section as a template. Protein expression, cell lysis and $\alpha$ Syn precipitation were performed as described for the WT full-length $\alpha$ Syn. The protein solution was solved using a cationic Resource-S column (Amersham Biosciences) and eluted with a linear gradient of $1 \mathrm{M} \mathrm{NaCl}$. Size exclusion chromatography and reverse phase chromatography purification steps were performed as described above. The fractions containing the isolated protein were lyophilized and stored as indicated for full-length $\alpha$ Syn. The protein concentration was determined by UV absorption at $280 \mathrm{~nm}$ using an extinction coefficient $\varepsilon 1 \%$ of 1.45 , calculated using the ExPASY tool ${ }^{46}$.

\section{$\underline{\text { Kinetics of fibril formation followed by thioflavin } \mathrm{T} \text { (ThT) }}$}

The formation of $\alpha$ Syn amyloid fibrils was studied by preparing solutions of $\alpha$ Syn $\left(0.5 \mathrm{mg} \mathrm{mL}^{-1}\right)$ in fibrillation buffer (50 mM Tris- $\mathrm{HCl} \mathrm{pH} 7.4,150 \mathrm{mM} \mathrm{NaCl})$ containing $10 \mu \mathrm{M}$ ThT in a total volume of $200 \mu \mathrm{L}$, which were placed in each well of a 96-well microplate, at $37^{\circ} \mathrm{C}$. The ThT fluorescence intensity was monitored using a HANdai Amyloid Burst Inducer (HANABI-2000) equipment, developed by our group in collaboration with CORONA ELECTRIC, Ibaraki, Japan, consisting of a transducer driving system, an acoustic-intensity measurement system, and a fluorescence measure system, in which a microplate reader was combined with a multichannel ultrasonication system that applies ultrasonic irradiation independently to each individual well of the microplate via a miniaturized ultrasonic resonator attached to each well. Ultrasonication was applied to accelerate amyloid formation at an optimized frequency of $30 \mathrm{kHz}^{31,47}$ in cycles of $300 \mathrm{~ms}$ of irradiation and $500 \mathrm{~ms}$ of quiescence. The kinetic parameters of fibril formation was determined by fitting the experimental data to empirical equations ${ }^{48}$.

\section{$\underline{\text { Patient information and tissue preparation. }}$}

Brain tissues from three MSA patients $(67.7 \pm 8.5$ years old, $M: F=3: 0)$ and three PD patients $(79.3$ \pm 2.8 years old, $\mathrm{M}: \mathrm{F}=2: 1$ ) were obtained from the autopsy series of the Brain Bank for Aging Research (BBAR) in the Tokyo Metropolitan Geriatric Hospital and Institute of Gerontology. The brains were processed according to the BBAR protocol. Briefly, half of the brain was sliced and frozen, 
and the other half was fixed in 20\% buffered formalin for 7-13 days, after which the representative anatomical areas were embedded in paraffin.

\section{Brain lysate preparation}

For this study, amygdala sections from the frozen side and amygdala sections from the formalin-fixed side were prepared. Amygdala slices of $100 \mathrm{mg}$ from the brains of PD and MSA patients were placed into Precellys Lysing tubes (M\&S Instruments, Osaka, Japan), resuspended in $1 \mathrm{~mL}$ of fibrillation buffer, and subjected to two cycles of high-speed shaking for $20 \mathrm{~s}$ in a Lysis and Homogenization System (Bertin Instruments, France). Then, the homogenates were transferred into Eppendorf tubes and centrifuged at $2000 \times \mathrm{g}$ for $2 \mathrm{~min}$ at room temperature. The concentration of total protein in the supernatant fractions was quantified using a MicroBCA Protein Assay Reagent Kit (Pierce), and the homogenates were aliquoted and stored at $-80{ }^{\circ} \mathrm{C}$ until use.

\section{Immunohistochemistry of brain samples.}

Immunohistochemistry was performed using a mouse monoclonal antibody against phosphorylated asynuclein (pSyn\#64; a gift from T. Iwatsubo, Japan), a Ventana BenchMark GX autostainer (Ventana Medical Systems, Tucson, AZ, USA), and an I-View Universal DAB Detection Kit (Roche, Basel, Switzerland). All slides were counterstained with haematoxylin to visualize neurons and other cells.

\section{$\underline{\text { Amplification of } \alpha \text { Syn aggregates from amygdala brain homogenates }}$}

A volume of amygdala brain homogenate was added to solutions of $\alpha$ Syn to reach a final concentration of $20 \mu \mathrm{g} \mathrm{mL}^{-1}$ total protein. Two hundred microliters of $0.5 \mathrm{mg} \mathrm{mL}^{-1}$ monomeric $\alpha$ Syn in the fibrillation buffer containing the amygdala lysate was placed into a 96-multiplate and subjected to ultrasonic irradiation to accelerate the formation of amyloid fibrils, as described above. The ThT fluorescence intensity was recorded as a function of time.

\section{$\underline{\text { Transmission electron microscopy (TEM) }}$}

TEM imaging was achieved on a Hitachi H-7650 transmission electron microscope (Hitachi, Tokyo) operated at $80 \mathrm{kV}$. Samples were diluted 1:10 with deionized water, and 10 $\mu \mathrm{L}$ of this solution was placed onto copper grids (400-mesh) covered with carbon-coated collodion film (Nisshin EM, Tokyo) and incubated for $1 \mathrm{~min}$ at room temperature. The samples were negatively stained with $10 \mu \mathrm{L}$ of a $1 \%(\mathrm{w} / \mathrm{v})$ solution of phosphotungstic acid (PTA), incubated for $1 \mathrm{~min}$, and finally washed again with $10 \mu \mathrm{L}$ of deionized water. The magnification working interval ranged from $5000 \times$ to $30000 \times$.

Proteinase K resistance (PKR) assay

$\alpha$ Syn fibrils $\left(0.5 \mathrm{mg} \mathrm{mL}^{-1}\right)$ in the fibrillation buffer were digested using proteinase $\mathrm{K}\left(1 \mu \mathrm{g} \mathrm{mL}{ }^{-1}\right.$, Roche) at $37{ }^{\circ} \mathrm{C}$ and $400 \mathrm{rpm}$ for different time intervals. To stop the reaction, the samples were incubated for 5 min at $95^{\circ} \mathrm{C}$, mixed with loading buffer (50 mM TRIS-HCl, pH 6.8, $4 \%$ SDS, $2 \% \beta$ - 
mercaptoethanol, $12 \%$ glycerol and $0.01 \%$ bromophenol blue) and incubated at $95{ }^{\circ} \mathrm{C}$ for additional 10 min. The digestion patterns were analyzed by SDS-PAGE followed by Coomassie Brilliant Blue staining. The first five products of digestion, namely B1 to B5, were employed for the analysis. The PKR Score has been established as the band intensity ratio between the bands B2 and B1 (B2/B1).

Analytical size-exclusion chromatography (SEC)

$\alpha$ Syn solutions were filtered through a $0.22 \mu \mathrm{m}$ size-pore membrane and loaded onto a Superdex 200 10/300 GL SEC column (GE Healthcare) coupled to an AKTA Explorer HPLC Instrument (Amersham Biosciences). The elution buffer was $50 \mathrm{mM}$ TRIS-HCl (pH 7.4) supplemented with $150 \mathrm{mM} \mathrm{NaCl}$. The flow rate was set to $0.5 \mathrm{~mL} \mathrm{~min}{ }^{-1}$. The protein absorption at 220, 250 and $280 \mathrm{~nm}$ was monitored. Analytical reverse phase chromatography

$\alpha$ Syn solutions in $5 \mathrm{mM} \mathrm{HCl}$ were filtered through a $0.22 \mu \mathrm{m}$ size-pore membrane and loaded onto a Cosmosil 5C4-AR-300 4.6ID x $150 \mathrm{~mm}$ analytical column (Nacalai-Tesque, Kyoto, Japan) coupled to a Gilson HPLC Unipoint System Instrument (Gilson, USA) with a $30 \%$ v/v acetonitrile in water buffer supplemented with $0.05 \% \mathrm{v} / \mathrm{v}$ trifluoroacetic acid. The sample was eluted using a 30-90 \% v/v gradient of acetonitrile. The flow rate was set to $0.5 \mathrm{~mL} \mathrm{~min}^{-1}$. The protein absorption at 220 and $280 \mathrm{~nm}$ was monitored.

\section{$\underline{\text { Analytical ultracentrifugation (AUC) }}$}

Sedimentation velocity (SV) experiments were performed at $20{ }^{\circ} \mathrm{C}$ using an Optima AUC (Beckman Coulter) analytical ultracentrifuge (Fullerton, CA, USA) equipped with an An-50Ti rotor using 12$\mathrm{mm}$ double-sector charcoal-filled epon centerpiece with sapphire windows. The protein concentrations were adjusted to absorbance values of 0.8 in the working buffer at $1-\mathrm{cm}$ path length. Sedimentation data were collected every $2 \mathrm{~min}$ at 40,000 rpm with a radial increment of $0.001 \mathrm{~cm}$ using absorbance optics. The detection wavelength was set at $230 \mathrm{~nm}$.

The distribution of the sedimentation coefficient was determined using the continuous $\mathrm{c}(\mathrm{s})$ distribution model in the program SEDFIT ${ }^{49}$. The range of the sedimentation coefficients for fitting was $0-15 \mathrm{~S}$, with a resolution of 300 . The partial specific volume of the sample, the buffer density, and the buffer viscosity were calculated by the program SEDNTERP ${ }^{50}$, respectively.

\section{Multidimensional NMR}

${ }^{1} \mathrm{H}-{ }^{15} \mathrm{~N}$ heterogenous single-quantum coherence (HSQC) NMR measurements were performed by using $100 \mu \mathrm{M}{ }^{15} \mathrm{~N}$-labelled $\alpha$ Syn dissolved in fibrillation buffer prepared in $\mathrm{H}_{2} \mathrm{O} / \mathrm{D}_{2} \mathrm{O}(9: 1$, v/v). The ${ }^{15} \mathrm{~N}$-labelled $\alpha$ Syn was expressed in M9 minimal media containing ${ }^{15} \mathrm{NH}_{4} \mathrm{Cl}$ and purified as described for the unlabeled protein. NMR spectra were acquired at $310 \mathrm{~K}$ on a Bruker Advance III $600 \mathrm{MHz}$ NMR spectrometer equipped with a QCI-P cryogenic probe (Bruker Biospin). The peak assignment at 
613

pH 7.4 was achieved employing the assignment data reported by El Turk et $a l^{51}$. Chemical shift perturbation (CSP) was calculated as follows:

$$
\Delta \delta=\sqrt{\Delta \delta_{H}^{2}+\left(\frac{1}{8} \Delta \delta_{N}\right)^{2}}
$$

where $\Delta \delta_{\mathrm{H}}$ and $\Delta \delta_{\mathrm{N}}$ are the chemical shift changes (in ppm) with respect to the $\mathrm{H}$ and $\mathrm{N}$ axes, respectively.

For backbone dynamics study, ${ }^{1} \mathrm{H}^{-15} \mathrm{~N}$ hetero NOE of ${ }^{15} \mathrm{~N} \alpha$ Syn were obtained by measured ${ }^{1} \mathrm{H}-{ }^{15} \mathrm{~N}$ HSQC spectra with and without ${ }^{1} \mathrm{H}$ saturation for 3 seconds, and then calculating the ratios of the intensities of the ${ }^{1} \mathrm{H}-{ }^{15} \mathrm{~N}$ peaks. The errors in hetero NOE values were estimated using root-meansquare value of the spectrum background noise.

All NMR spectra were processed with Topspin (Bruker Biospin), NMRPipe ${ }^{52}$ and NMRFAMsparky ${ }^{53}$.

\section{$\underline{\text { Structural Model Selection }}$}

576 disordered conformations of $\alpha$ Syn from the Protein Ensemble Database (https://proteinensemble.org/) (PED ID: PED00024 ${ }^{34}$ ) were used to measure deviations between the chemical shifts predicted based on conformations and the shifts observed in this study. Predicted shifts and root-mean-square (RMS) deviations between the predicted and experimental shifts were calculated using SPARTA $+{ }^{54}$. The ${ }^{1} \mathrm{H}$ - and ${ }^{15} \mathrm{~N}$ - chemical shifts of aSyn in 0,150 and $500 \mathrm{mM} \mathrm{NaCl}$ were used for the calculations.

\section{Circular dichroism (CD) spectroscopy}

CD measurements were performed using a Jasco J820 spectrophotometer at $37{ }^{\circ} \mathrm{C}$. Quartz cuvettes of $1 \mathrm{~cm}$ were utilized. The employed protein concentration was $5.0 \mathrm{mg} \mathrm{mL}^{-1}$ in fibrillation buffer. Spectra were recorded in 350-250 $\mathrm{nm}$ (near-UV CD) ranges and reported as the mean residue ellipticity ( $[\theta]_{\mathrm{MRW}}$, deg $\left.\mathrm{cm}^{2} \mathrm{dmol}^{-1}\right)$ after subtracting the baseline.

\section{$\underline{\text { Statistical analysis }}$}

We used Statistical Package for the Social Sciences 23.0J software (IBM Japan, Tokyo, Japan) to perform statistical analysis. For the statistical analysis of 2 groups, the paired or unpaired t-test was used as described in the figure legends. For more than 3 groups, we used one-way analysis of variance (ANOVA) with the Bonferroni/Dunn post hoc test. In all experiments, data were expressed as the mean $\pm \mathrm{SD}$, and a $p$ value of $<0.05$ was considered to indicate a statistically significant difference between 2 groups $(* p<0.05$. ** $p<0.001, * * * p<0.0001)$.

\section{Ethical Statement}


The collection of autopsied human tissues and their use for this study were approved by the Ethics Committee of Osaka University Graduate School of Medicine, and written informed consent was obtained from the patients' next of kin. Experimental procedures involving human subjects were conducted in conformance with the principles expressed in the Declaration of Helsinki.

\section{References}

1. Spillantini, M. G. et al. Alpha-synuclein in Lewy bodies. Nature 388, 839-840 (1997).

2. Spillantini, M. G., Crowther, R. A., Jakes, R., Hasegawa, M. \& Goedert, M. $\alpha$-Synuclein in filamentous inclusions of Lewy bodies from Parkinson's disease and dementia with Lewy bodies. Proc. Natl. Acad. Sci. U. S. A. (1998). doi:10.1073/pnas.95.11.6469

3. Gai, W. P., Power, J. H. T., Blumbergs, P. C. \& Blessing, W. W. Multiple-system atrophy: A new $\alpha$-synuclein disease? Lancet (1998). doi:10.1016/S0140-6736(05)79256-4

4. Araki, K. et al. Synchrotron FTIR micro-spectroscopy for structural analysis of Lewy bodies in the brain of Parkinson's disease patients. Sci. Rep. 5, 1-8 (2015).

5. Araki, K. et al. Parkinson's disease is a type of amyloidosis featuring accumulation of amyloid fibrils of $\alpha$-synuclein. Proc. Natl. Acad. Sci. U. S. A. 116, 17963-17969 (2019).

6. Araki, K. et al. The secondary structural difference between Lewy body and glial cytoplasmic inclusion in autopsy brain with synchrotron FTIR micro-spectroscopy. Sci. Rep. 10, 1-6 (2020).

7. Van der Perren, A. et al. The structural differences between patient-derived $\alpha$-synuclein strains dictate characteristics of Parkinson's disease, multiple system atrophy and dementia with Lewy bodies. Acta Neuropathol. (2020). doi:10.1007/s00401-020-02157-3

8. Shahnawaz, M. et al. Discriminating $\alpha$-synuclein strains in Parkinson's disease and multiple system atrophy. Nature 578, 273-277 (2020).

9. Schweighauser, M. et al. Structures of $\alpha$-synuclein filaments from multiple system atrophy. Nature 585, 464-469 (2020).

10. Prusiner, S. B. et al. Evidence for $\alpha$-synuclein prions causing multiple system atrophy in humans with parkinsonism. Proc. Natl. Acad. Sci. U. S. A. (2015). doi:10.1073/pnas.1514475112

11. Yamasaki, T. R. et al. Parkinson's disease and multiple system atrophy have distinct synuclein seed characteristics. J. Biol. Chem. (2019). doi:10.1074/jbc.RA118.004471

12. Peng, C. et al. Cellular milieu imparts distinct pathological $\alpha$-synuclein strains in $\alpha$ synucleinopathies. Nature 1 (2018). doi:10.1038/s41586-018-0104-4

13. Fusco, G. et al. Structural basis of membrane disruption and cellular toxicity by $\alpha$-synuclein oligomers. Science (80-. ). 358, 1440-1443 (2017).

14. Suzuki, G. et al. $\alpha$-synuclein strains that cause distinct pathologies differentially inhibit proteasome. Elife (2020). doi:10.7554/eLife.56825

15. Hayakawa, H. et al. Structurally distinct $\alpha$-synuclein fibrils induce robust parkinsonian pathology. Mov. Disord. 1-12 (2019). doi:10.1002/mds.27887

16. Peelaerts, W., Bousset, L., Baekelandt, V. \& Melki, R. a-Synuclein strains and seeding in Parkinson's disease, incidental Lewy body disease, dementia with Lewy bodies and multiple system atrophy: similarities and differences. Cell Tissue Res. 373, 195-212 (2018).

17. Ikenaka, K., Suzuki, M., Mochizuki, H. \& Nagai, Y. Lipids as Trans-Acting Effectors for $\alpha-$ 
Synuclein in the Pathogenesis of Parkinson's Disease. Front. Neurosci. 13, 1-9 (2019).

18. Brundin, P. \& Melki, R. Prying into the Prion Hypothesis for Parkinson's Disease. $J$. Neurosci. 37, 9808-9818 (2017).

19. Bousset, L. et al. Structural and functional characterization of two alpha-synuclein strains. Nat. Commun. 4, 2575 (2013).

20. Peelaerts, W. et al. $\alpha$-Synuclein strains cause distinct synucleinopathies after local and systemic administration. Nature 522, 340-344 (2015).

21. Bertoncini, C. W. et al. Release of long-range tertiary interactions potentiates aggregation of natively unstructured $\alpha$-synuclein. Proc. Natl. Acad. Sci. U. S. A. 102, 1430-1435 (2005).

22. Kessler, J. C., Rochet, J. C. \& Lansbury, P. T. The N-terminal repeat domain of $\alpha$-synuclein inhibits $\beta$-sheet and amyloid fibril formation. Biochemistry 42, 672-678 (2003).

23. Zhou, W. et al. Methionine oxidation stabilizes non-toxic oligomers of $\alpha$-synuclein through strengthening the auto-inhibitory intra-molecular long-range interactions. Biochim. Biophys. Acta - Mol. Basis Dis. 1802, 322-330 (2010).

24. Izawa, Y. et al. Role of C-terminal negative charges and tyrosine residues in fibril formation of ??-synuclein. Brain Behav. 2, 595-605 (2012).

25. Ranjan, P. \& Kumar, A. Perturbation in Long-Range Contacts Modulates the Kinetics of Amyloid Formation in $\alpha$-Synuclein Familial Mutants. ACS Chem. Neurosci. 8, 2235-2246 (2017).

26. Furukawa, K. et al. Isoelectric point-amyloid formation of $\alpha$-synuclein extends the generality of the solubility and supersaturation-limited mechanism. Curr. Res. Struct. Biol. 2, 35-44 (2020).

27. Morar, A. S., Olteanu, A., Young, G. B. \& Pielak, G. J. Solvent-induced collapse of $\alpha-$ synuclein and acid-denatured cytochrome c. Protein Sci. 10, 2195-2199 (2008).

28. Edwards, A. J. \& Reid, D. Introduction to NMR of Proteins. Curr. Protoc. Protein Sci. 19, Unit 17.5 (2000).

29. Ulrih, N. P., Barry, C. H. \& Fink, A. L. Impact of Tyr to Ala mutations on $\alpha$-synuclein fibrillation and structural properties. Biochim. Biophys. Acta - Mol. Basis Dis. 1782, 581-585 (2008).

30. Kakuda, K. et al. Ultrasonication-based rapid amplification of $\alpha$-synuclein aggregates in cerebrospinal fluid. Sci. Rep. 9, 6001 (2019).

31. Nakajima, K. et al. Optimized sonoreactor for accelerative amyloid-fibril assays through enhancement of primary nucleation and fragmentation. Ultrason. Sonochem. 73, 105508 (2021).

32. Atarashi, R. et al. Ultrasensitive human prion detection in cerebrospinal fluid by real-time quaking-induced conversion. Nat. Med. 17, 175-178 (2011).

33. Nakagaki, T., Nishida, N. \& Satoh, K. Development of $\alpha$-Synuclein Real-Time QuakingInduced Conversion as a Diagnostic Method for $\alpha$-Synucleinopathies. Front. Aging Neurosci. 0, 637 (2021).

34. Allison, J. R., Varnai, P., Dobson, C. M. \& Vendruscolo, M. Determination of the free energy landscape of $\alpha$-synuclein using spin label nuclear magnetic resonance measurements. $J$. Am. Chem. Soc. (2009). doi:10.1021/ja904716h

35. Stephens, A. D. et al. Extent of N-terminus exposure of monomeric alpha-synuclein determines its aggregation propensity. Nat. Commun. 11, (2020).

36. Bhattacharyya, D. \& Bhunia, A. Gut-Brain axis in Parkinson's disease etiology: The role of 
lipopolysaccharide. Chem. Phys. Lipids 235, 105029 (2021).

37. Raetz, C. R. H. BIOCHEMISTRY OF ENDOTOXINS. Annu. Rev. Biochem. 59, 129-170 (1990).

38. Barkleit, A. et al. Coordination of uranium(vi) with functional groups of bacterial lipopolysaccharide studied by EXAFS and FT-IR spectroscopy. Dalt. Trans. 40, 9868-9876 (2011).

39. Oueslati, A. Implication of Alpha-Synuclein Phosphorylation at S129 in Synucleinopathies: What Have We Learned in the Last Decade? J. Parkinsons. Dis. 6, 39-51 (2016).

40. Schapira, A. H. V. Calcium dysregulation in Parkinson's disease. Brain 136, 2015-2016 (2013).

41. Doherty, C. P. A. et al. A short motif in the N-terminal region of $\alpha$-synuclein is critical for both aggregation and function. Nat. Struct. Mol. Biol. 27, 249-259 (2020).

42. Mazzulli, J. R. et al. Gaucher disease glucocerebrosidase and $\alpha$-synuclein form a bidirectional pathogenic loop in synucleinopathies. Cell (2011). doi:10.1016/j.cell.2011.06.001

43. Li, B. et al. Cryo-EM of full-length $\alpha$-synuclein reveals fibril polymorphs with a common structural kernel. Nat. Commun. 9, 3609 (2018).

44. Guerrero-Ferreira, R. et al. Cryo-EM structure of alpha-synuclein fibrils. Elife 7, 1-18 (2018).

45. Narhi, L. et al. Both familial Parkinson's disease mutations accelerate alpha-synuclein aggregation. J. Biol. Chem. 274, 9843-9846 (1999).

46. Gasteiger, E. et al. ExPASy: The proteomics server for in-depth protein knowledge and analysis. Nucleic Acids Res. 31, 3784-3788 (2003).

47. Nakajima, K. et al. Drastic acceleration of fibrillation of insulin by transient cavitation bubble. Ultrason. Sonochem. 36, 206-211 (2017).

48. Arosio, P., Knowles, T. P. J. \& Linse, S. On the lag phase in amyloid fibril formation. Phys. Chem. Chem. Phys. 17, 7606-7618 (2015).

49. Schuck, P. Size-distribution analysis of macromolecules by sedimentation velocity ultracentrifugation and Lamm equation modeling. Biophys. J. 78, 1606-1619 (2000).

50. Laue \& M., T. Computer-aided interpretation of analytical sedimentation data for proteins. in Analytical ultracentrifugation in biochemistry and polymer science. 90-125 (Royal Society of Chemistry, 1992).

51. El Turk, F. et al. Exploring the role of post-translational modifications in regulating $\alpha-$ synuclein interactions by studying the effects of phosphorylation on nanobody binding. Protein Sci. 27, 1262-1274 (2018).

52. Delaglio, F. et al. NMRPipe: A multidimensional spectral processing system based on UNIX pipes. J. Biomol. NMR 6, 277-293 (1995).

53. Lee, W., Tonelli, M. \& Markley, J. L. NMRFAM-SPARKY: Enhanced software for biomolecular NMR spectroscopy. Bioinformatics 31, 1325-1327 (2015).

54. Shen, Y. \& Bax, A. SPARTA+: A modest improvement in empirical NMR chemical shift prediction by means of an artificial neural network. J. Biomol. NMR (2010). doi:10.1007/s10858-010-9433-9 


\section{Acknowledgments}

We would like to thank Shizuka Sonoda and Miki Yoshida for the protein expression and purification and the excellent technical support received. CA thanks JSPS (postdoctoral fellowship P16388) for the financial support.

Funding: This work was supported by JSPS KAKENHI Grant JP16H06277, JP18H02741; JST CREST Grant Numbers JPMJCR18H4 and JPMJCR17H6; JSPS Core-to-Core Program A Advance Research Networks; AMED Grant Numbers JP18dm0107103, JP19dm0207001, JP20dm0107106, JP20dm0107158, JP20km0405206, JP20am0101110 and SENTAN16809202; and the Ministry of Education, Culture, Sports, Science and Technology Grant Number 17H06352. The Brain Mapping by Integrated Neuroethologies for Disease Studies (Brain/MINDS) from Japan Agency for Medical Research and development, AMED JP18dm0207020. CA was funded by JSPS KAKENHI Grant 16F16388.

Author contributions: CA, KI, YG, and HM designed the experiments. CA, KI, MS, TM, KY, KN, CJC, KN, GB, KT, YY, JD, TM, MM, KK, MH, YK, SM and YM performed the experiments. $\mathrm{CA}$ and $\mathrm{KI}$ analyzed the data. $\mathrm{CA}$ and $\mathrm{KI}$ wrote the original draft. MS, YK, SN, $\mathrm{KB}, \mathrm{HO}, \mathrm{YN}, \mathrm{YK}, \mathrm{SU}, \mathrm{YM}, \mathrm{YG}$ and $\mathrm{HM}$ reviewed the manuscript.

Competing interests: Authors declare no conflicts of interest.

Data and materials availability: All data is available in the main text or the supplementary materials.

Dedications: We would like to express our deep gratitude to Professor Kazuhiro Ikenaka for his support, precious advices, and discussions, especially for the glycolipids section. 
804

805

Figures and Tables

A

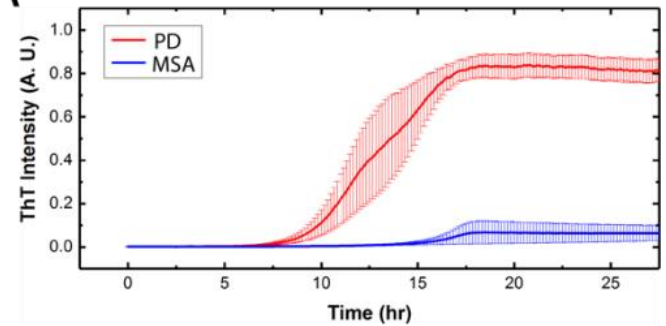

C
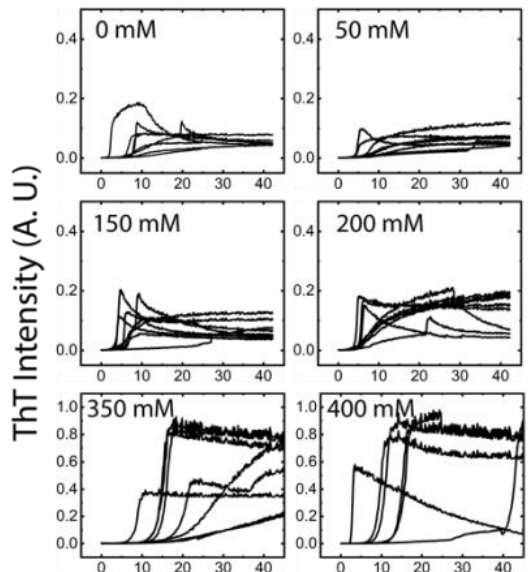

Time $(\mathrm{h})$

E
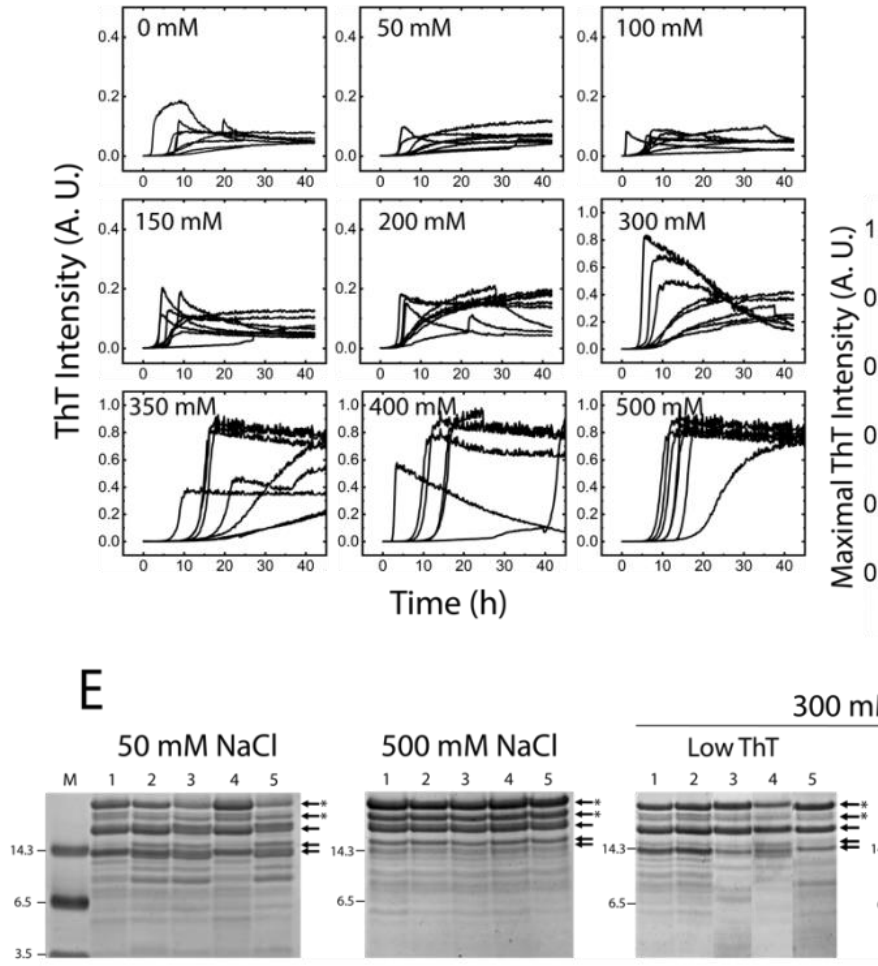

F

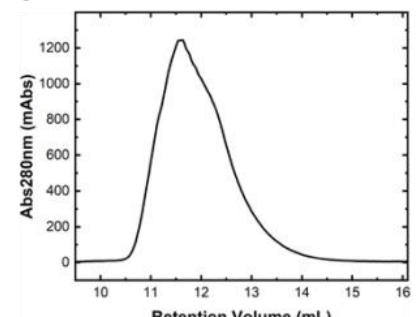

Glor

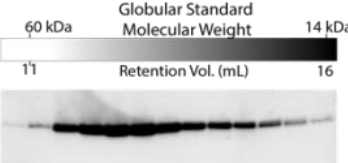

G

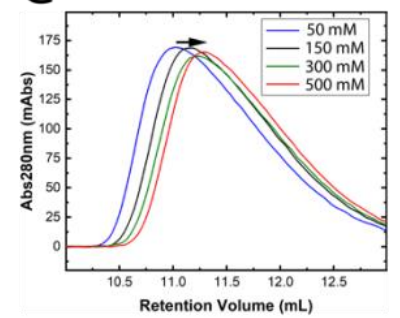

806

807
B
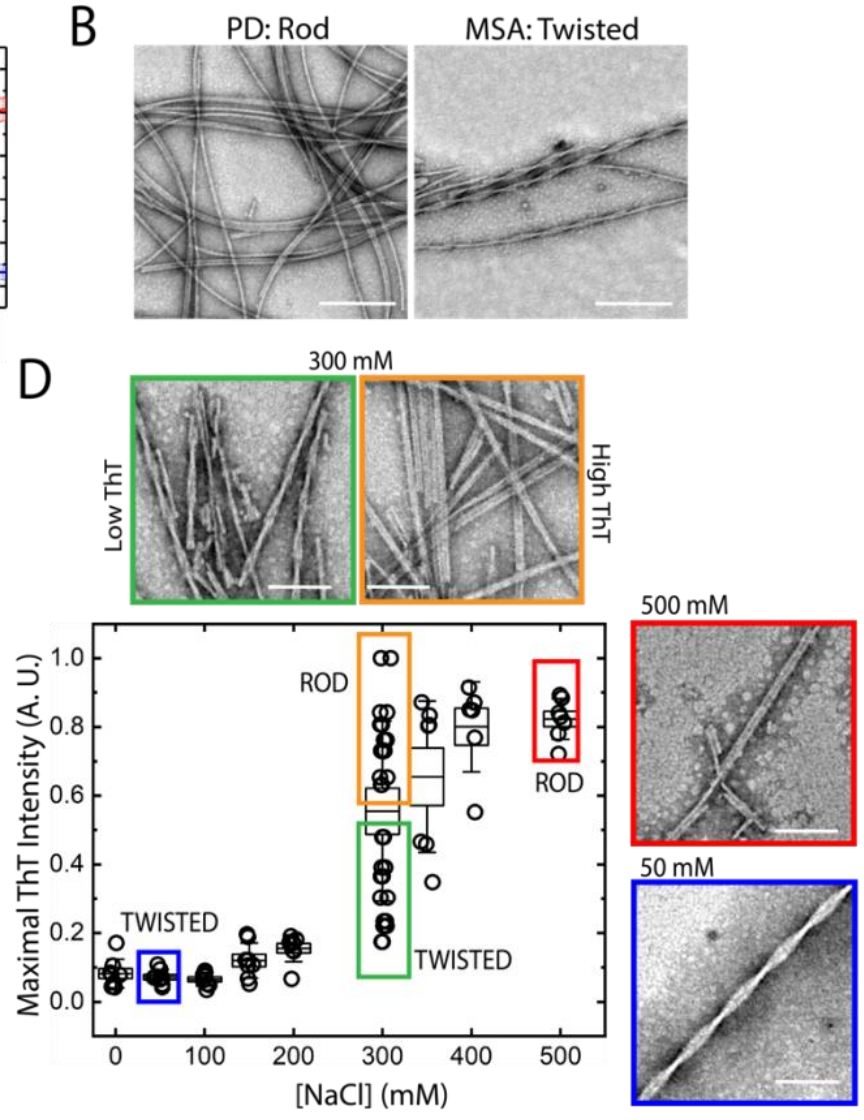

$0 \mathrm{mM} \mathrm{NaCl}$

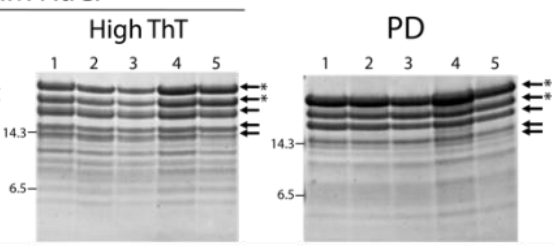

MSA

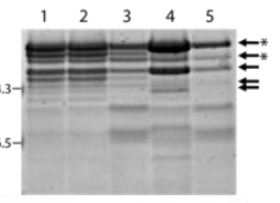

$\mathrm{H}_{1.2}$
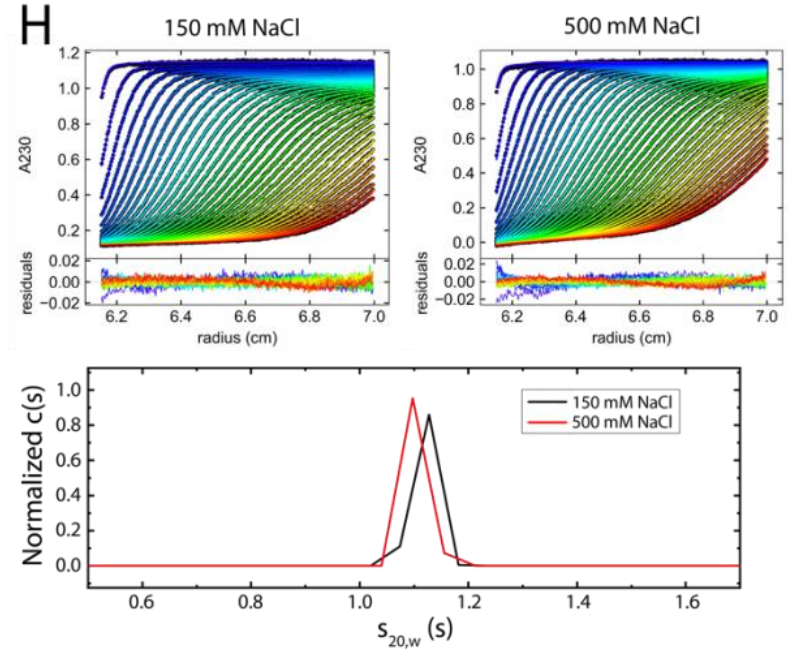
Figure 1. $\alpha$ Syn fibril morphology is modulated by the conformational state of the monomeric $\alpha$ Syn. (A) PD-(red) and MSA-(blue)-amygdala-seeded fibril amplification monitored by ThT intensity (mean \pm s.e.m. of three different patients). (B) TEM visualization of fibrils amplified from the brain of patients. (C) ThT-monitored $\alpha$ Syn fibril formation at different concentrations of $\mathrm{NaCl}$. (D) Maximal ThT intensity values of fibrils created under the indicated concentration of $\mathrm{NaCl}$. Representative amyloid fibrils obtained at $50 \mathrm{mM}$ (low ThT, blue square), $300 \mathrm{mM}$ (low ThT, green square; high ThT, orange square) and $500 \mathrm{mM}$ (red square) were visualized by TEM. (Bar scale: 200 $\mathrm{nm}$ ). (E) From left to right, proteinase-K resistance assay of five replicates (numbers 1-5) of fibrils produced at $50 \mathrm{mM}, 500 \mathrm{mM}$, created at $300 \mathrm{mM} \mathrm{NaCl}$ with maximal low- or high-ThT values, and amplified from PD and MSA brain of patients. Arrows indicate five selected bands subjected to intensity analysis. Bands marked with $(*)$ denote the most relevant differences. Molecular weight markers (kDa) are shown on the left of each panel. (F) A solution of $\alpha$ Syn in $50 \mathrm{mM}$ TRIS pH 7.4 supplemented with $300 \mathrm{mM} \mathrm{NaCl}$ was solved by SEC (upper) and the fractions obtained were analyzed by Western Blotting (Anti-Syn211, lower). (G) Representative chromatograms of Solutions of monomeric $\alpha$ Syn at different concentrations of $\mathrm{NaCl}$ solved by Size Exclusion Chromatography. (H) Analytical Ultracentrifugation Sedimentation Velocity measurements of solutions of monomeric $\alpha$ Syn in the presence of $150 \mathrm{mM}$ (upper-left) and $500 \mathrm{mM}$ (upper-right) $\mathrm{NaCl}$. The sedimentation coefficient values $\left(s_{20, w}\right)$ were determined to be $1.136 \pm 0.012 \mathrm{~S}$ and $1.092 \pm 0.011 \mathrm{~S}$ for the monomers at 150 

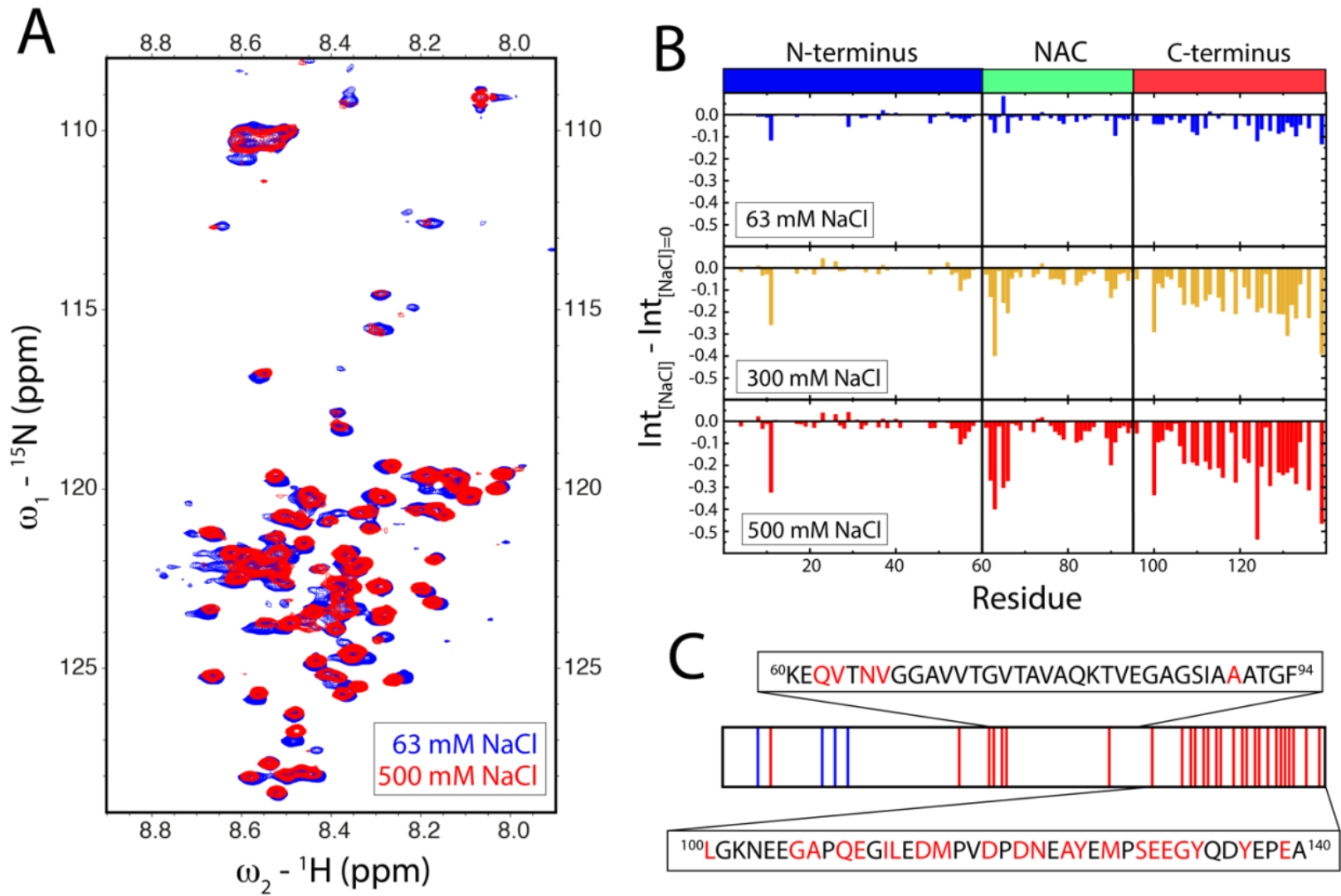
domain and NAC region. (A) ${ }^{1} \mathrm{H}^{-15} \mathrm{~N}$ HSQC spectra of solutions of $\alpha$ Syn supplemented with $63 \mathrm{mM}$ (blue) and $500 \mathrm{mM}$ (red) $\mathrm{NaCl}$. (B) Peak intensity difference analysis of the spectra of $\alpha$ Syn of the amide $\mathrm{N}-\mathrm{H}$ nuclei recorded at $63 \mathrm{mM}, 300 \mathrm{mM}$, and $500 \mathrm{mM} \mathrm{NaCl}$, employing the spectrum obtained at $0 \mathrm{mM} \mathrm{NaCl}$ as a reference. Gray bars represent the residues without signal assignments. (C) Residues that exhibit the most relevant changes in peak intensity, marked as red lines. The blue lines represent residues with slight increases in peak intensity. 
A

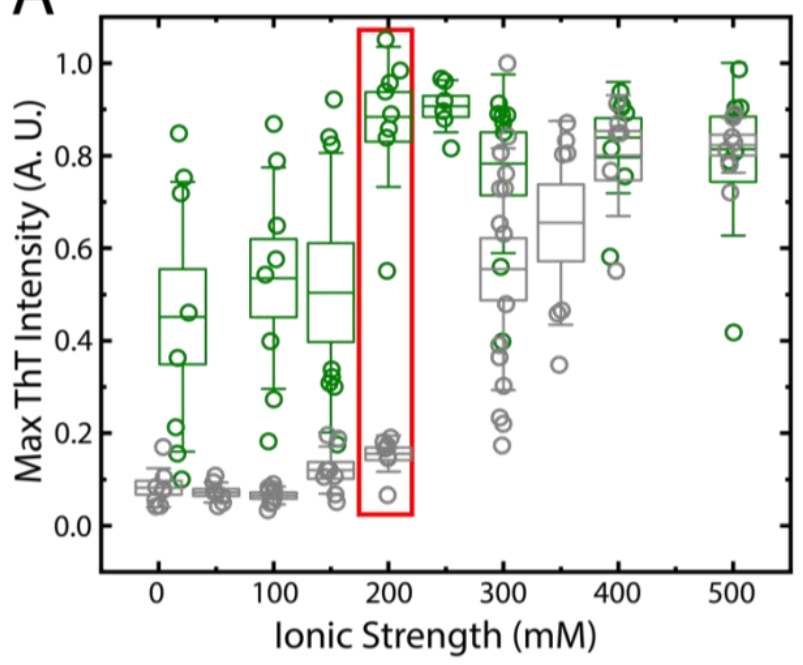

E

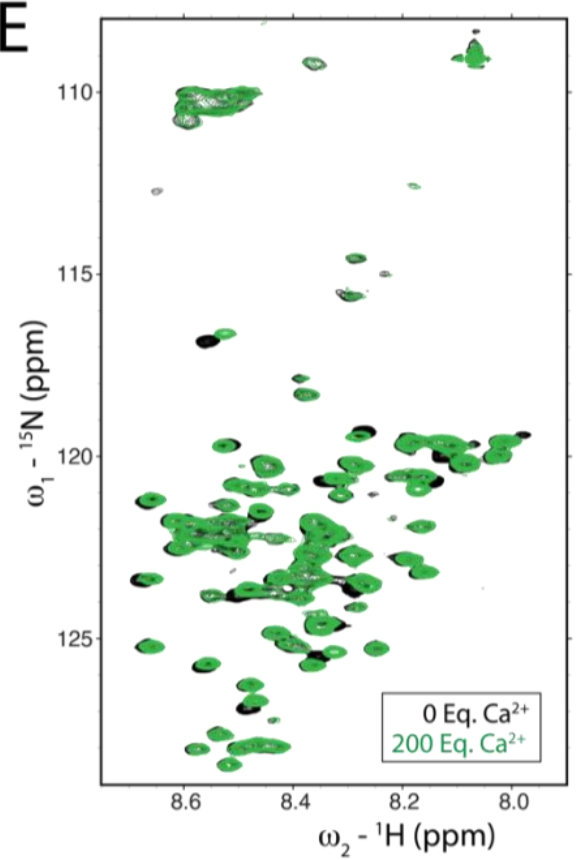

B
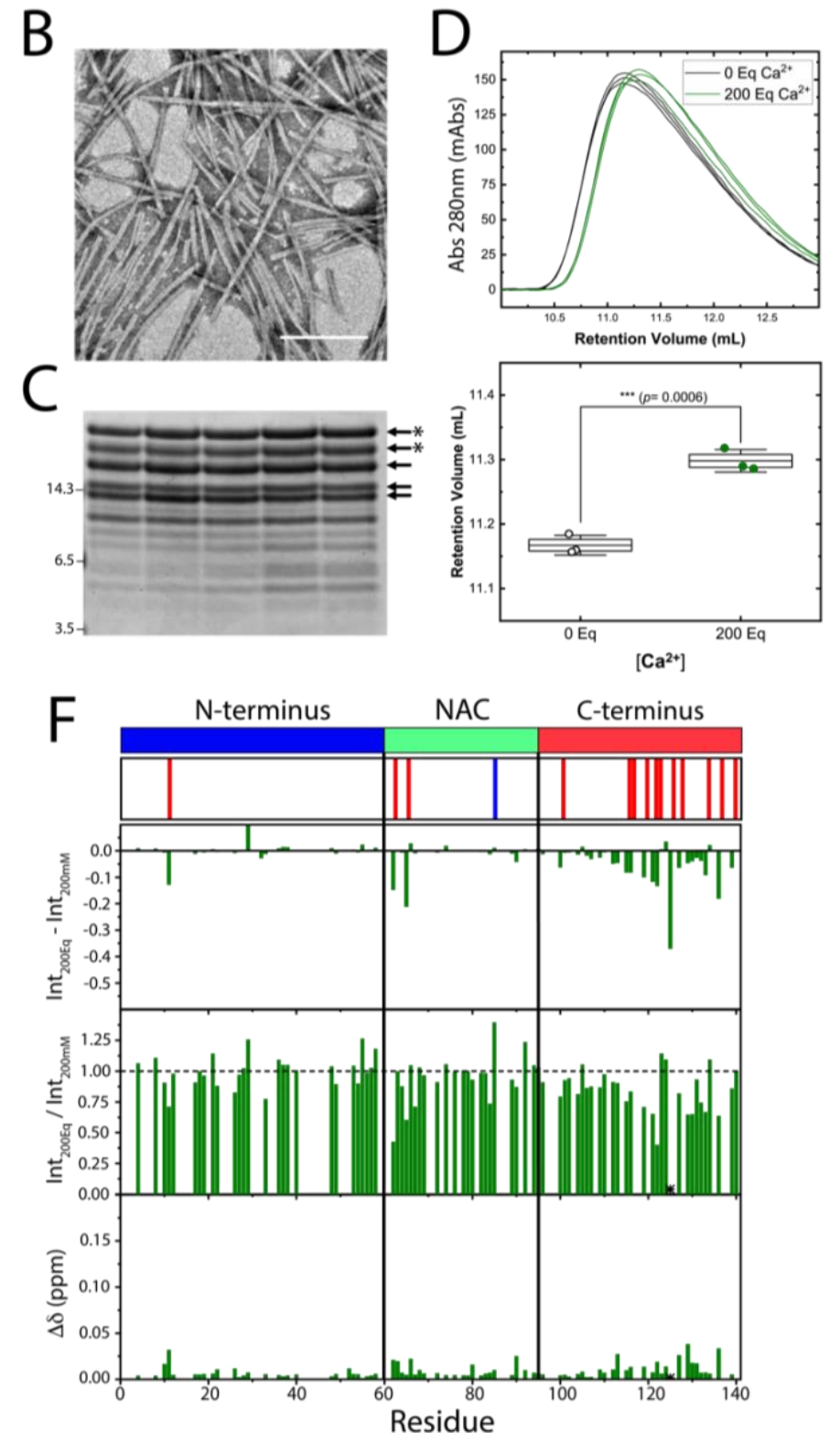

Figure 3. $\mathrm{Ca}^{2+}$ enhances the formation of $\alpha \mathrm{Syn}$ rod-like fibrils through the compaction of monomers. (A) Maximal ThT intensity values of fibrils created under the indicated ionic strength in the presence (green) or absence (gray) of $200 \mathrm{Eq}$. $\mathrm{Ca}^{2+}$. (The values of maximal ThT in the absence of $\mathrm{Ca}^{2+}$ are the same as those shown in Figure 1.D. They were inserted here for comparative purposes). (B) TEM visualization of $\alpha$ Syn fibrils created at an ionic strength of $200 \mathrm{mM}$ in the presence of 200 Eq. $\mathrm{Ca}^{2+}$ (Bar scale: $200 \mathrm{~nm}$ ). (C) PKR assay of representative fibrils obtained in the presence of 200 Eq. $\mathrm{Ca}^{2+}$ at an ionic strength of $200 \mathrm{mM}$. Arrows indicate five selected bands subjected to intensity analysis. Bands marked with $(*)$ denote the most relevant differences. Molecular weight markers (kDa) are shown on the left of each panel. (D) Representative chromatograms of solutions of monomeric $\alpha$ Syn in the presence or absence of $200 \mathrm{Eq} . \mathrm{Ca}^{2+}$, at an ionic strength of $200 \mathrm{mM}$, solved by Size Exclusion Chromatography (top). Retention volume values of $\alpha$ Syn monomers analyzed by SEC in the presence or absence of $200 \mathrm{Eq}$. $\mathrm{Ca}^{2+}$ at an ionic strength of $200 \mathrm{mM}$ (bottom). (E) ${ }^{1} \mathrm{H}^{-}{ }^{15} \mathrm{~N} \mathrm{HSQC}$ spectra of solutions of $\alpha$ Syn supplemented with or without $200 \mathrm{Eq} \mathrm{Ca}^{2+}$ at an ionic strength of 200 mM. (F) Analysis of intensity difference (top), ratio (middle) and Chemical Shift Perturbation (bottom) of the N-H nuclei employing the spectrum obtained at in the absence of $\mathrm{Ca}^{2+}$, at $200 \mathrm{mM}$ of ionic strength, as reference. Gray bars represent the residues without signal assignments. $\left({ }^{*}\right.$ Represents signals that disappeared in the condition tested but that are present in the reference spectrum) 

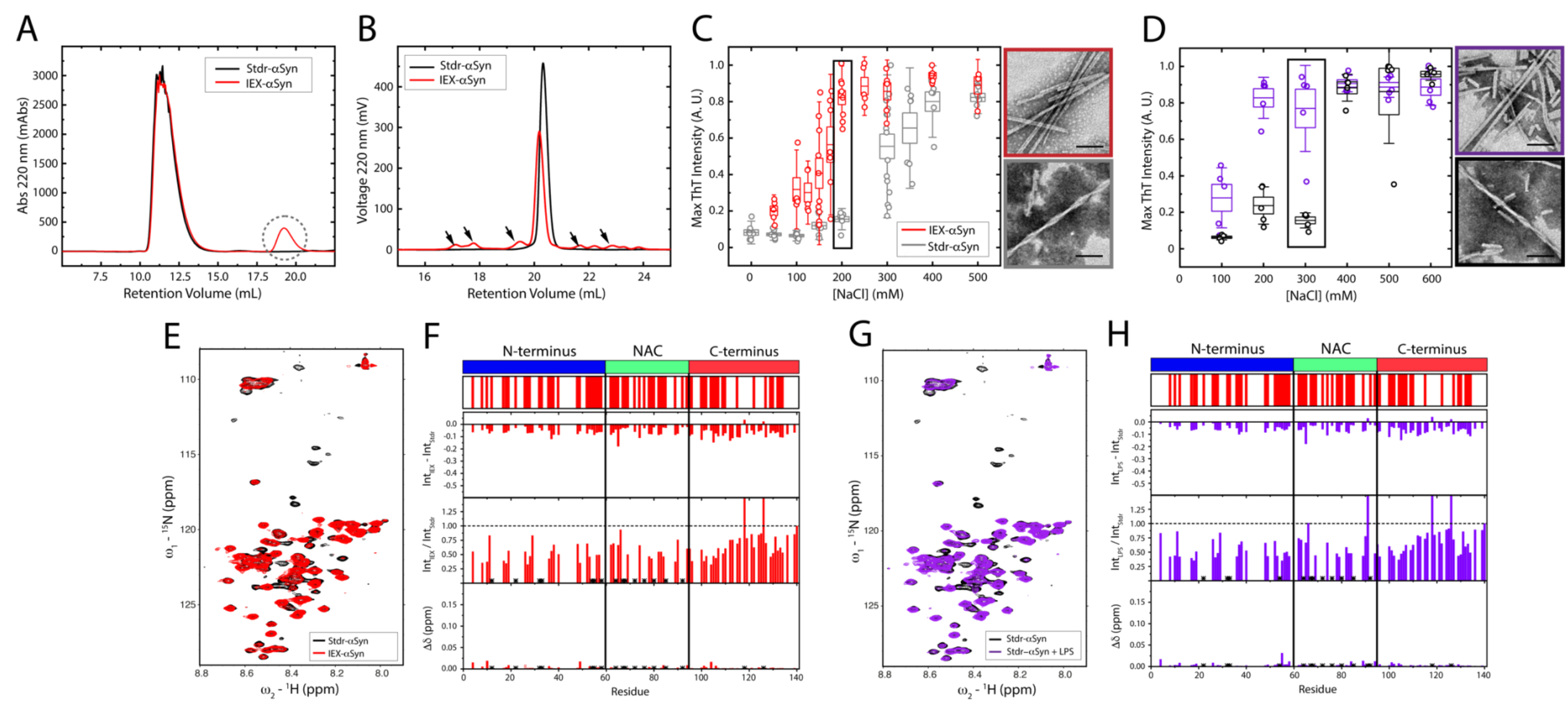

$\mathrm{F}$

G
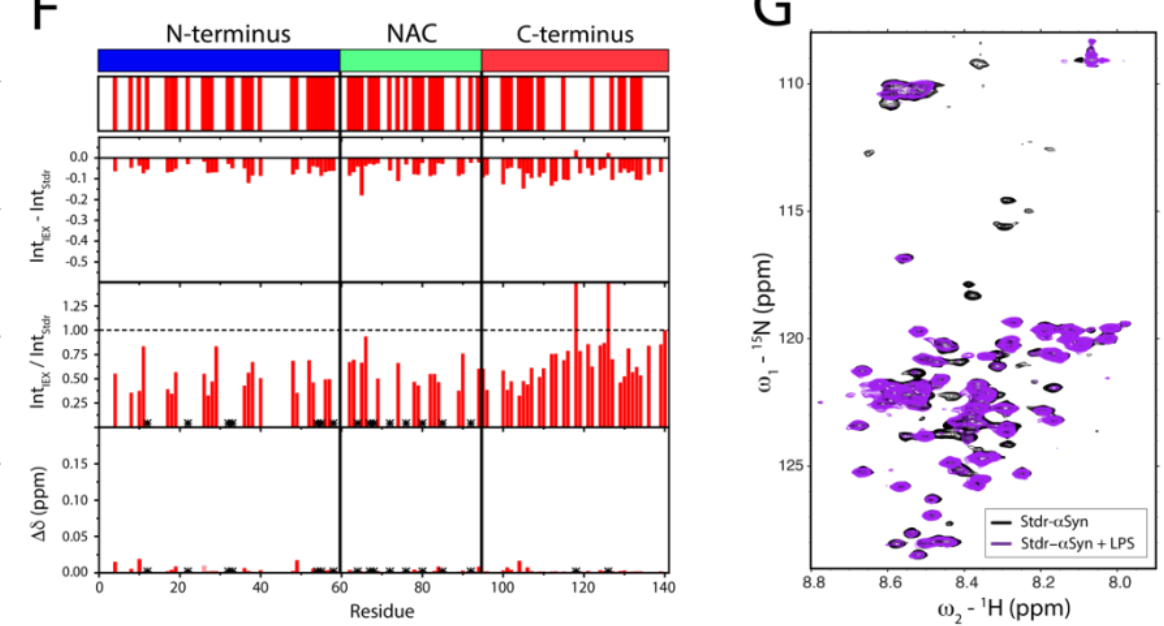

$\mathrm{H}$

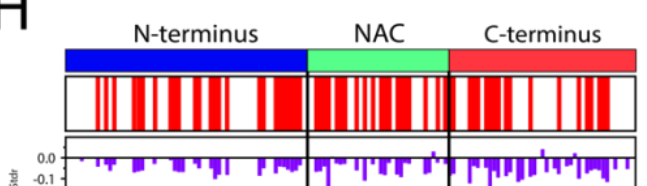

I

'MDVFMKGLSKAKEGV's

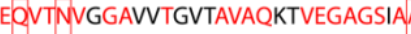

${ }^{50} K E Q V T T N G G A V V T G V T A V A Q K T V E G A G S I A A{ }^{\circ}$

${ }^{105}$ EGAPQEGILEDMPVDPDNEAYEMMSSEEG YQDYEPEA ${ }^{140}$

LPS 'MDVFMKGLSKAKEGV's

${ }^{105}$ EGAPQEGILEDMPVDPDNEAYEMPSEEGYQDYEPEA ${ }^{140}$

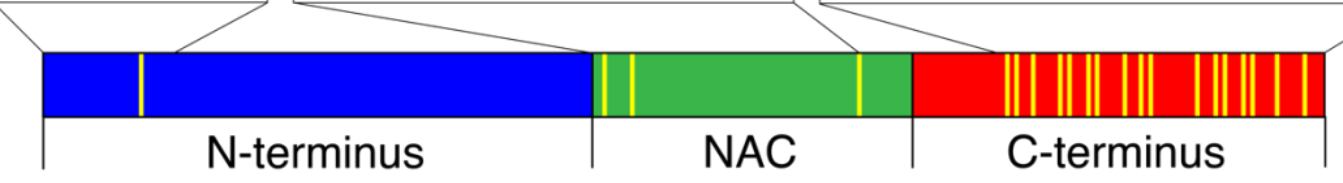


Figure 4. Bacterial components present in the purification of recombinant proteins affect the conformational state of the monomeric $\alpha$ Syn. IEX- $\alpha$ Syn and HPLC- $\alpha$ Syn were solved by analytical size exclusion chromatography (A) and analytical reverse phase chromatography (B). (C) Left: Maximal ThT intensity values of fibrils created using IEX- $\alpha$ Syn (red) and HPLC- $\alpha$ Syn (gray, taken from Fig. 1.D for comparison). Right: TEM visualization of representative fibrils obtained at $200 \mathrm{mM} \mathrm{NaCl}$ using IEX- $\alpha$ Syn (upper) and HPLC- $\alpha$ Syn (lower). (D) Left: Maximal ThT intensity values of fibrils created using HPLC- $\alpha$ Syn in the presence (purple) or absence (black) of LPS. Right: TEM visualization of representative fibrils obtained at $300 \mathrm{mM} \mathrm{NaCl}$ using HPLC- $\alpha$ Syn in the presence (upper) or absence (lower) of LPS. (E) ${ }^{1} \mathrm{H}-{ }^{15} \mathrm{~N}$ HSQC spectra of solutions of IEX- $\alpha$ Syn (red) and HPLC- $\alpha$ Syn (black) at an ionic strength of $200 \mathrm{mM}$. (F) Analysis of intensity difference (top), ratio (middle) and chemical shift perturbation (bottom) of the N-H nuclei of IEX- $\alpha$ Syn employing the spectrum of HPLC- $\alpha$ Syn as reference. Gray bars represent the residues without signal assignments. (* Represents signals that disappeared in the condition tested but that are present in the reference spectrum) (G) ${ }^{1} \mathrm{H}^{-15} \mathrm{~N}$ HSQC spectra of solutions of HPLC- $\alpha$ Syn in the presence (purple) or absence (black) of LPS at an ionic strength of $200 \mathrm{mM}$. (H) Analysis of intensity difference (top), ratio (middle) and chemical shift perturbation (bottom) of the N-H nuclei of HPLC- $\alpha$ Syn treated with LPS employing the spectrum of HPLC- $\alpha$ Syn without LPS as reference. Gray bars represent the residues without signal assignments. ( ${ }^{*}$ Represents signals that disappeared in the condition tested but that are present in the reference spectrum) (I) Alignment of the residues affected by the compaction of $\alpha$ Syn under the conditions indicated in the schematic diagram. The method to determine the affected residues is shown in the Fig. S6. 
bioRxiv preprint doi: https://doi.org/10.1101/2022.02.10.479831; this version posted February 10, 2022. The copyright holder for this preprint (which was not certified by peer review) is the author/funder, who has granted bioRxiv a license to display the preprint in perpetuity. It is made available under aCC-BY-NC-ND 4.0 International license.

A

Full-Length aSyn

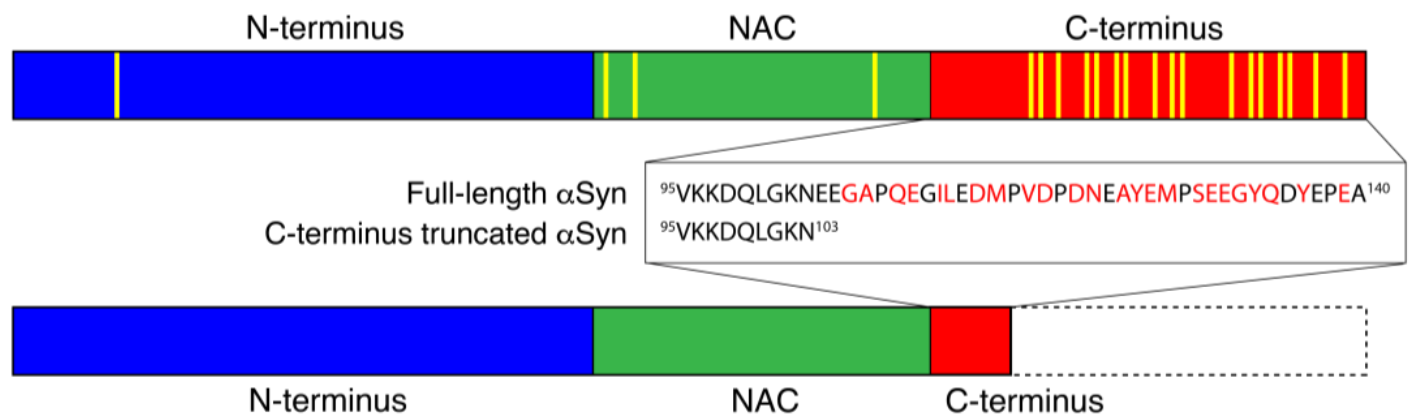

\section{C-terminus truncated $\alpha$ Syn}

B

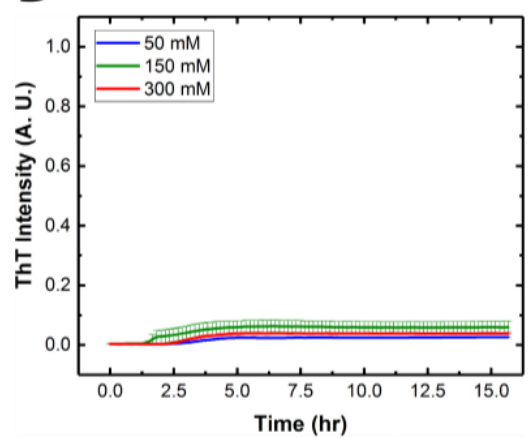

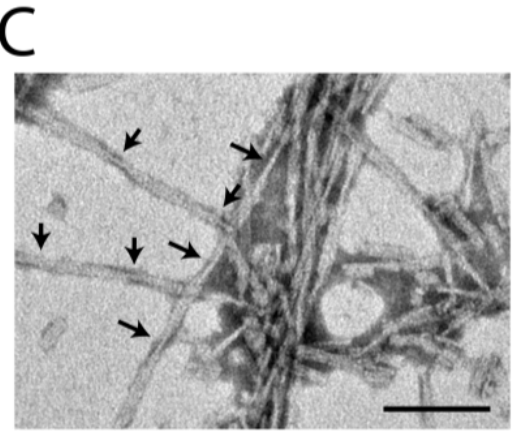

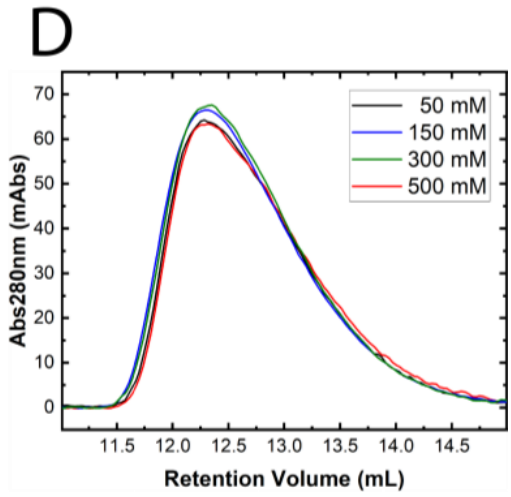

886

Figure 5. N103t-C terminus truncated $\alpha$ Syn does not adopt the compact conformation. (A) Schematic representation of the truncated form N103t, in which the region detected to be relevant for the compaction of the monomers has been removed. (B) ThT-monitored fibril formation of Cterminus truncated N103t $\alpha$ Syn at 50, 150 and $300 \mathrm{mM} \mathrm{NaCl}$. (C) TEM visualization of N103t $\alpha$ Syn fibrils generated at $300 \mathrm{mM} \mathrm{NaCl}$. (D) Representative chromatograms of solutions of N103t $\alpha$ Syn at different concentrations of $\mathrm{NaCl}$ solved by Size Exclusion Chromatography. 
bioRxiv preprint doi: https://doi.org/10.1101/2022.02.10.479831; this version posted February $10,2022$. The copyright holder for this

preprint (which was not certified by peer review) is the author/funder, who has granted bioRxiv a license to display the preprint in perpetuity. It is made available under aCC-BY-NC-ND 4.0 International license.

Low lonic Strength

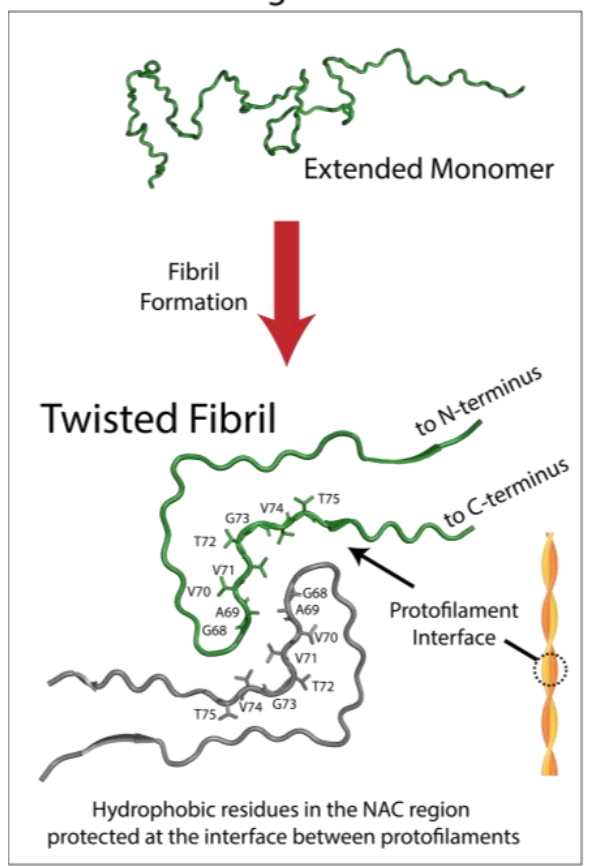

Compaction of monomers (i.e. High lonic Strength)

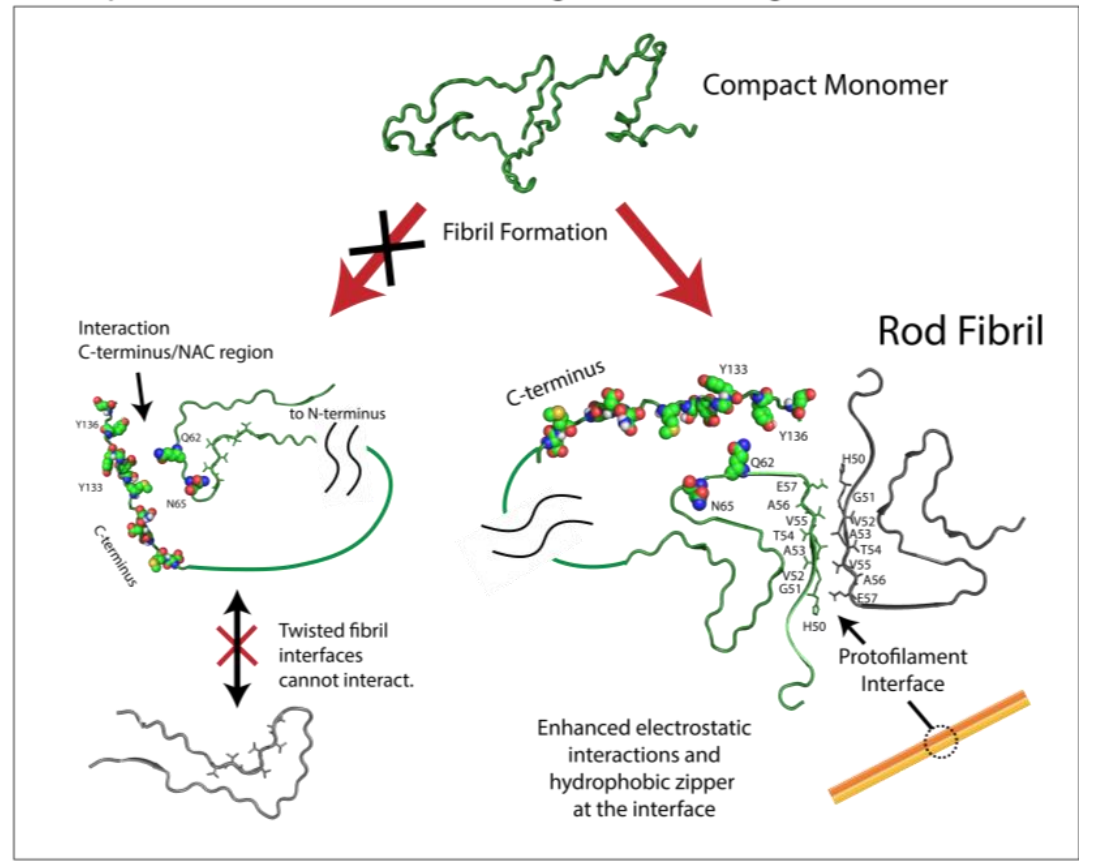

Figure 6. The conformational state of the monomeric $\alpha$ Syn provides differences between fibril morphologies. The initial state of monomeric $\alpha \mathrm{Syn}$ and the factors that induce affect the Cterminus domain determine the final morphology of the fibrils. In the brain, this phenomenon could trigger the development of PD or MSA. 